\title{
Northcott's theorem on heights \\ II. The quadratic case
}

by

\author{
Wolfgang M. Schmidt (Boulder, Colo.)
}

1. Introduction. The distribution of algebraic points in projective space $\mathbb{P}^{n}(A)$, where $A$ is the field of algebraic numbers, is best described in terms of their height. When $K$ is an algebraic number field and $P$ a point in $\mathbb{P}^{n}(K)$, let $H_{K}(P)$ be the multiplicative field height as defined in [8], [11], [12], [13] or [14]. When $P=\left(\alpha_{0}: \ldots: \alpha_{n}\right)$ lies in $\mathbb{P}^{n}(A)$, let $K=\mathbb{Q}(P)$ be the field obtained from $\mathbb{Q}$ by adjoining the ratios $\alpha_{i} / \alpha_{j}\left(0 \leq i, j \leq n ; \alpha_{j} \neq 0\right)$, and set $\mathcal{H}(P)=H_{K}(P)$. Note that $\mathcal{H}(P)$ is the $d$ th power of the absolute height $H(P)$ as defined in the literature, where $d=\operatorname{deg} \mathbb{Q}(P)$.

Given a field $K$, let $N(K, n, X)$ be the number of points $P \in \mathbb{P}^{n}(K)$ with $H_{K}(P) \leq X$. Given $d$, let $\mathcal{N}(d, n, X)$ be the number of points $P \in \mathbb{P}^{n}(A)$ with $\operatorname{deg} \mathbb{Q}(P)=d$ and $\mathcal{H}(P) \leq X$.

Schanuel [11] had proved an asymptotic formula

$$
\begin{aligned}
N(K, n, X)= & c_{1}(K, n) X^{n+1} \\
& + \begin{cases}O(X \log X) & \text { when } d=n=1, \\
O_{K n}\left(X^{n+1-(1 / d)}\right) & \text { otherwise. }\end{cases}
\end{aligned}
$$

The constant $c_{1}(K, n)$ was explicitly given by Schanuel; like all constants in this paper, it is positive. Further $d=\operatorname{deg} K$, and the constant implicit in $O_{K n}(\ldots)$ depends on $K$ and $n$ only. On the other hand, the quantity $\mathcal{N}(d, n, X)$ is finite by Northcott's Theorem [10] but its estimation is more difficult. In the first part [13] of the present series we showed that for given $d, n$ and $X>X_{0}(d, n)$,

$$
c_{2}(d, n) X^{\max (d+1, n+1)}<\mathcal{N}(d, n, X)<c_{3}(d, n) X^{d+n} .
$$

(In fact, we dealt with the more general situation where the condition $[\mathbb{Q}(P)$ : $\mathbb{Q}]=d$ was replaced by $[k(P): k]=d$, where $k$ is a given algebraic number field.) In the present paper we will obtain more information in the case when $d=2$.

Supported in part by NSF grant DMS-9108581. 
Let $N^{\prime}(K, n, X)$ be the number of points $P \in \mathbb{P}^{n}(K)$ with $\mathbb{Q}(P)=K$ and $H_{K}(P) \leq X$. (Note that $\mathcal{H}(P)=H_{K}(P)$ for such points.) It is easily seen that $N^{\prime}(K, n, X)$ satisfies the same asymptotic formula (1.1) as $N(K, n, X)$. Since

$$
\mathcal{N}(d, n, X)=\sum_{K} N^{\prime}(K, n, X),
$$

where the sum is over all number fields $K$ of degree $d$, it is tempting to take the sum over the right hand side of (1.1). However, in order to do so, one needs to know the implied constants in $O_{K n}(\ldots)$. (One also needs information on the collection of all fields of given degree $d$; this information is readily available only for $d=2$, when the fields are parametrized by their discriminant.)

In the present paper we will obtain a more precise version of (1.1) for quadratic fields $K$. Our work will also lead to a more explicit form of a classical asymptotic formula of Dirichlet on ideals with bounded norm in a given quadratic number field. (This formula was later extended to arbitrary fields by Dedekind.)

Let $K$ be a quadratic number field with discriminant $\Delta$, class number $h$, and with $w$ roots of unity. In the case when $K$ is real, so that $\Delta>0$, let $\varepsilon>1$ be the fundamental unit. Set

$$
\begin{gathered}
R= \begin{cases}1 & \text { when } \Delta<0, \\
\log \varepsilon & \text { when } \Delta>0,\end{cases} \\
\lambda= \begin{cases}2 \pi & \text { when } \Delta<0, \\
4 & \text { when } \Delta>0 .\end{cases}
\end{gathered}
$$

Finally, for $X>0$, let $Z(K, X)$ be the number of nonzero integral ideals $\mathfrak{a}$ in $K$ with norm $\mathfrak{N}(\mathfrak{a}) \leq X$. Dirichlet's asymptotic formula says that when $K$ is fixed and $X \rightarrow \infty$, then

$$
Z(K, X) \sim \frac{\lambda h R}{w|\Delta|^{1 / 2}} X .
$$

It is easily seen that the error term here is $O_{K}\left(X^{1 / 2}\right)$. In fact, the exponent $1 / 2$ can be reduced, but we will not be concerned with this here. Rather we will estimate the implied constant in $O_{K}$.

THEOREM 1.

$$
Z(K, X)=\frac{\lambda h R}{w|\Delta|^{1 / 2}} X+O\left(\left(X h R \log ^{+}(h R)\right)^{1 / 2}\right) .
$$

Here the implied constant in $O(\ldots)$ is absolute, and $\log ^{+} x=$ $\max (1, \log x)$. In fact, all the constants which will occur in the sequel in $O(\ldots)$ or in $\ll$ will depend only on occasional parameters $n, m, l, \sigma, \alpha, \delta$, but will be independent of the field $K$. 
Schanuel's constant $c_{1}(K, n)$ occurring in (1.1), in the case of a quadratic field $K$, is given by

$$
c_{1}(K, n)=\frac{\nu h R}{w \zeta_{K}(n+1)}\left(\frac{\lambda}{|\Delta|^{1 / 2}}\right)^{n+1},
$$

where $\zeta_{K}$ is the Dedekind zeta function of $K$ and where

$$
\nu= \begin{cases}1 & \text { when } \Delta<0 \\ n+1 & \text { when } \Delta>0\end{cases}
$$

We now introduce

$$
c_{1}^{*}(K, n)=|\Delta|^{-n / 2}\left(h R \log ^{+}(h R)\right)^{1 / 2} .
$$

Theorem 2. For a quadratic field $K$,

$$
N^{\prime}(K, n, X)=c_{1}(K, n) X^{n+1}+O\left(c_{1}^{*}(K, n) X^{n+(1 / 2)}\right) .
$$

This leads also to an estimate for $N(K, n, X)$. For the points counted by $N(K, n, X)$ but not by $N^{\prime}(K, n, X)$ are points $P$ with $\mathbb{Q}(P)=\mathbb{Q}$, i.e., with $P \in \mathbb{P}^{n}(\mathbb{Q})$ and $H_{K}(P)=H_{\mathbb{Q}}(P)^{2} \leq X$. Therefore

$$
N(K, n, X)=N^{\prime}(K, n, X)+N\left(\mathbb{Q}, n, X^{1 / 2}\right)=N^{\prime}(K, n, X)+O\left(X^{(n+1) / 2}\right) .
$$

Write

$$
\mathcal{N}(2, n, X)=\mathcal{N}^{-}(2, n, X)+\mathcal{N}^{+}(2, n, X),
$$

where $\mathcal{N}^{-}(2, n, X), \mathcal{N}^{+}(2, n, X)$ is the number of points $P \in \mathbb{P}^{n}(A)$ with $\operatorname{deg} \mathbb{Q}(P)=2$ and $\mathcal{H}(P) \leq X$, and where the discriminant $\Delta(\mathbb{Q}(P))$ is $<0$ or $>0$, respectively.

TheOREM 3. When $n \geq 3$, then

$$
\mathcal{N}^{ \pm}(2, n, X)=c_{5}^{ \pm}(n) X^{n+1}+O\left(X^{n+(1 / 2)}\right)
$$

with certain constants $c_{5}^{+}(n), c_{5}^{-}(n)$ defined in Section 8 . Here and below, the relations hold with superscript + throughout, or superscript - throughout. Further when $n=2$,

$$
\mathcal{N}^{ \pm}(2,2, X)=c_{6}^{ \pm} X^{3} \log X+O\left(X^{3} \sqrt{\log X}\right)
$$

with

and when $n=1$,

$$
c_{6}^{+}=\frac{48}{\zeta(3)^{2}}, \quad c_{6}^{-}=\frac{4 \pi^{2}}{\zeta(3)^{2}},
$$

$$
\mathcal{N}^{ \pm}(2,1, X)=c_{7}^{ \pm} X^{3}+O\left(X^{2} \log X\right)
$$

with

$$
c_{7}^{+}=\frac{40}{9 \zeta(3)}, \quad c_{7}^{-}=\frac{32}{9 \zeta(3)} .
$$


The theorem shows that for $d=2$, the lower bounds in (1.2) are near the truth. We expect this to be true in general. In fact Gao Xia will soon publish results for $d>2$.

Next, we consider nonzero quadratic forms

$$
f\left(x_{0}, \ldots, x_{n}\right)=\sum_{0 \leq i \leq j \leq n} a_{i j} x_{i} x_{j}
$$

with rational coefficients. The form is called decomposable if it is the product of two linear forms with algebraic coefficients. When $f$ is decomposable, say $f=l l^{\prime}$ with $l(\boldsymbol{x})=\sum_{i=0}^{n} \alpha_{i} x_{i}, l^{\prime}(\boldsymbol{x})=\sum_{i=0}^{n} \alpha_{i}^{\prime} x_{i}$, then by unique factorization the (unordered) pair of points $P=\left(\alpha_{0}: \ldots: \alpha_{n}\right), P^{\prime}=$ $\left(\alpha_{0}^{\prime}: \ldots: \alpha_{n}^{\prime}\right)$ in $\mathbb{P}^{n}(A)$ is uniquely determined by $f$. We have $\mathbb{Q}(P)=$ $\mathbb{Q}\left(P^{\prime}\right)=K(f)$, say, with $K(f)$ either a quadratic or the rational field.

Let $\mathcal{Z}(n, X)$ be the number of decomposable quadratic forms with coefficients $a_{i j} \in \mathbb{Z}$ having $\left|a_{i j}\right| \leq X(0 \leq i \leq j \leq n)$. We write

$$
\mathcal{Z}(n, X)=\mathcal{Z}^{-}(n, X)+\mathcal{Z}^{+}(n, X)+\mathcal{Z}^{0}(n, X),
$$

where $\mathcal{Z}^{-}, \mathcal{Z}^{+}, \mathcal{Z}^{0}$ respectively count only those forms for which $K(f)$ is imaginary quadratic, real quadratic, or the rational field. Since every form in 1 or 2 variables is decomposable, the interesting cases are when $n \geq 2$.

THEOREM 4. We have

$$
\begin{aligned}
& \mathcal{Z}^{ \pm}(2, X)=c_{8}^{ \pm}(2) X^{3} \log X+O\left(X^{3} \sqrt{\log X}\right), \\
& \mathcal{Z}^{ \pm}(n, X)=c_{8}^{ \pm}(n) X^{n+1}+O\left(X^{n+(1 / 2)}\right) \quad \text { when } n \geq 3 .
\end{aligned}
$$

On the other hand, for $n \geq 2$,

$$
\mathcal{Z}^{0}(n, X)=c_{8}^{0}(n) X^{n+1} \log X+O\left(X^{n+1}\right) .
$$

In particular, $\mathcal{Z}(n, X) \sim c_{9}(n) X^{n+1} \log X$ for $n \geq 2$. It is somewhat surprising that when $n \geq 3$, the number $\mathcal{Z}^{0}(n, X)$ is of larger order of magnitude than $\mathcal{Z}^{-}(n, X)$ or $\mathcal{Z}^{+}(n, X)$. Our proof will imply fairly explicit values for the constants $c_{8}^{ \pm}(n)$.

The form $f$ could also be written as

$$
f=\sum_{i, j=0}^{n} b_{i j} x_{i} x_{j}
$$

with $b_{i j}=b_{j i}$. The form $f$ is decomposable precisely when the symmetric matrix $\left(b_{i j}\right)$ has rank $\leq 2$. Therefore $\mathcal{Z}(n, X)$ may be interpreted as the number of symmetric $(n+1) \times(n+1)$-matrices with rank $\leq 2$ such that $b_{i i} \in \mathbb{Z},\left|b_{i i}\right| \leq X$, and $2 b_{i j} \in \mathbb{Z}, 2\left|b_{i j}\right| \leq X$ for $i \neq j$. Of particular interest is the number $\mathcal{Z}(2, X)$, which counts symmetric $3 \times 3$-matrices. By a slight generalization of our method it would be possible to obtain a complete 
analog of Theorem 4 for the number $\mathcal{Z}_{1}(n, X)=\mathcal{Z}_{1}^{-}(n, X)+\mathcal{Z}_{1}^{+}(n, X)+$ $\mathcal{Z}_{1}^{0}(n, X)$, say, where $\mathcal{Z}_{1}(n, X)$ is the number of symmetric matrices $\left(b_{i j}\right)$ of rank $\leq 2$ and order $n+1$ with $b_{i j} \in \mathbb{Z},\left|b_{i j}\right| \leq X(0 \leq i, j \leq n)$. Many other variations of Theorem 4 could be given.

For the number $\mathcal{Z}_{2}(n, X)$ of singular $(n+1) \times(n+1)$-matrices $\left(b_{i j}\right)$ (not necessarily symmetric) with $b_{i j} \in \mathbb{Z},\left|b_{i j}\right| \leq X$, Katznelson [7] gave an asymptotic formula $\mathcal{Z}_{2}(n, X) \sim c_{10}(n) X^{n^{2}+n} \log X$, so that in particular $\mathcal{Z}_{2}(2, X) \sim c_{10}(3) X^{3} \log X$.

There are two directions in which one could try to generalize Theorem 4. On the one hand, one could consider decomposable forms of degree $d$ (rather than $d=2$ ); this leads essentially to questions (formulated at the beginning) on heights of points of degree $d$. On the other hand, one could consider symmetric matrices of rank $\leq d\left(^{1}\right)$.

In the appendix we will treat certain sums over $L$-series which will be needed in the proofs of Theorems 3 and 4 .

2. The number of lattice points in certain regions. Let $\Lambda$ be a lattice in $\mathbb{R}^{l}$ of determinant $\operatorname{det} \Lambda$, and let $\mathcal{S}$ be a compact set in $\mathbb{R}^{l}$ of volume $V(\mathcal{S})$. Under suitable conditions, the cardinality of $\Lambda \cap \mathcal{S}$ is about $V(\mathcal{S}) / \operatorname{det} \Lambda$. To make this precise, one needs information both on $\Lambda$ and on $\mathcal{S}$. The "shape" of $\Lambda$ is roughly described by the successive minima $\lambda_{1} \leq \ldots \leq \lambda_{l}$ of $\Lambda$, as defined by Minkowski. Here $\lambda_{i}$ is least such that $\Lambda$ contains $i$ linearly independent points with Euclidean norm $\leq \lambda_{i}$. We have

$$
c_{11}(l) \leq \lambda_{1} \ldots \lambda_{l} / \operatorname{det} \Lambda \leq c_{12}(l),
$$

according to Minkowski. (See, e.g., Cassels [2, Ch. VIII] or Siegel [17, Theorem 16].) $\mathcal{S}$ will be said to be of class $m$ if every line intersects $\mathcal{S}$ in the union of at most $m$ intervals and single points, and if the same is true of the projections of $\mathcal{S}$ on any linear subspace. In particular, $\mathcal{S}$ is convex when it is of class 1 .

Lemma 1. Suppose $\mathcal{S}$ is of class $m$, and it lies in the compact ball of radius $r$ and center $\mathbf{0}$. Let $\Lambda$ be a lattice, and $N$ the cardinality of $\Lambda \cap \mathcal{S}$. Then if

$$
\lambda_{l-1} \leq r
$$

we have

$$
N=\frac{V(\mathcal{S})}{\operatorname{det} \Lambda}+O\left(\frac{\lambda_{l} r^{l-1}}{\operatorname{det} \Lambda}\right)
$$

$\left({ }^{1}\right)$ Added in proof. For general matrices of fixed rank, see Y. Katznelson, Integral matrices of fixed rank (preprint). For symmetric matrices of fixed rank, see A. Eskin and Y. Katznelson, Singular symmetric matrices, Duke Math. J., to appear. 
The implicit constant in $O(.$.$) depends only on l, m$, in agreement with the convention made in the introduction.

Proof. There are independent lattice points $\boldsymbol{g}_{1}, \ldots, \boldsymbol{g}_{l}$ with $\boldsymbol{g}_{i} \in \lambda_{i} \mathcal{B}$ $(i=1, \ldots, l)$, where $\mathcal{B}$ is the closed unit ball. In fact (see $[2$, p. 135 , Lemma 8]), there is a basis $\boldsymbol{b}_{1}, \ldots, \boldsymbol{b}_{l}$ of $\Lambda$ with $\boldsymbol{b}_{i} \in i \lambda_{i} \mathcal{B}(i=1, \ldots, l)$. Let $\tau$ be the linear map with $\tau\left(\boldsymbol{b}_{i}\right)=\boldsymbol{e}_{i}$, where $\boldsymbol{e}_{i}=(0, \ldots, 1, \ldots, 0)$ (with 1 in the $i$ th component). Thus $\tau(\Lambda)=\mathbb{Z}^{l}$ and $\tau(\mathcal{B})=\mathcal{E}$, where $\mathcal{E}$ is an ellipsoid of volume $V(\mathcal{E})=V(\mathcal{B}) / \operatorname{det} \Lambda$. Now $\boldsymbol{e}_{i} \in i \lambda_{i} \mathcal{E}$, therefore $\left(i \lambda_{i}\right)^{-1} \boldsymbol{e}_{i} \in \mathcal{E}(i=1, \ldots, l)$, so that $\mathcal{E}$ has major axes of lengths $a_{1} \leq \ldots \leq a_{l}$ with $a_{i} \gg \lambda_{l-i+1}^{-1}(i=1, \ldots, l)$. Therefore, the orthogonal projection of $\mathcal{E}$ on any $i$-dimensional subspace has volume

$$
\begin{aligned}
& \ll a_{l-i+1} \ldots a_{l} \ll\left(a_{1} \ldots a_{l-i}\right)^{-1} V(\mathcal{E}) \ll \lambda_{i+1} \ldots \lambda_{l} V(\mathcal{E}) \\
& \ll \lambda_{i+1} \ldots \lambda_{l} / \operatorname{det} \Lambda .
\end{aligned}
$$

Now $N$ is the cardinality of $\mathbb{Z}^{n} \cap \mathcal{T}$ where $\mathcal{T}=\tau(\mathcal{S})$. According to Davenport [3],

$$
|N-V(\mathcal{T})| \ll \max _{\mathcal{T}^{\prime}} V\left(\mathcal{T}^{\prime}\right),
$$

where the maximum is over the orthogonal projections $\mathcal{T}^{\prime}$ of $\mathcal{T}$ on the coordinate planes of dimension $<l$, and where the volume of the 0 -dimensional projection is understood to be 1 . Here we have used the fact that $\mathcal{T}$ is of class $m$. Note that $V(\mathcal{T})=V(\mathcal{S}) / \operatorname{det} \Lambda$. Moreover, $\mathcal{S} \subset r \mathcal{B}$, therefore $\mathcal{T} \subset r \mathcal{E}$, and any $i$-dimensional projection $\mathcal{T}_{i}^{\prime}$ has

$$
V\left(\mathcal{T}_{i}^{\prime}\right) \ll r^{i} \lambda_{i+1} \ldots \lambda_{l} / \operatorname{det} \Lambda \leq \lambda_{l} r^{l-1} / \operatorname{det} \Lambda
$$

by (2.3), (2.2). The lemma follows.

We now give a variation on Lemma 1 valid in $\mathbb{R}^{2}$.

Lemma 2. Suppose $\mathcal{S} \subset \mathbb{R}^{2}$ is of class $m$, and contains the origin. Suppose it lies in the compact disc of radius $r$ and center $\mathbf{0}$. Let $\Lambda \subset \mathbb{R}^{2}$ be a lattice, and $N^{\prime}$ the number of nonzero lattice points in $\mathcal{S}$. Then

$$
N^{\prime}=V(\mathcal{S}) / \operatorname{det} \Lambda+O\left(r / \lambda_{1}\right) .
$$

Note that we do not stipulate a condition (2.2).

Proof. When $r \geq \lambda_{1}$, the assertion follows from the preceding lemma, since $N-N^{\prime}=1 \leq r / \lambda_{1}$ in this case. When $r<\lambda_{1}$, there is no nonzero lattice point in $\mathcal{S}$, so that $N^{\prime}=0$. Further $V(\mathcal{S}) / \operatorname{det} \Lambda \ll r^{2} / \lambda_{1} \lambda_{2}<r / \lambda_{1}$, since $r<\lambda_{1} \leq \lambda_{2}$.

Lemma 3. Let $\mathcal{S} \subseteq \mathbb{R}^{2 n+2}$ where $n \geq 1$. Suppose that $\mathcal{S}$ is of class $m$ and contained in the compact ball of radius $r$ and center $\mathbf{0}$. Write points $\boldsymbol{x} \in \mathbb{R}^{2 n+2}$ as $\boldsymbol{x}=\left(\boldsymbol{x}_{0}, \ldots, \boldsymbol{x}_{n}\right)$ with each $\boldsymbol{x}_{i} \in \mathbb{R}^{2}$. Let $\Lambda$ be a lattice in $\mathbb{R}^{2}$ 
with minima $\lambda_{1}, \lambda_{2}$. Then the number $N^{*}$ of points $\boldsymbol{x} \in \mathcal{S}$ such that each $\boldsymbol{x}_{i} \in \Lambda(i=0, \ldots, n)$, and $\boldsymbol{x}_{0}, \ldots, \boldsymbol{x}_{n}$ span $\mathbb{R}^{2}$, has

$$
N^{*}=\frac{V(\mathcal{S})}{(\operatorname{det} \Lambda)^{n+1}}+O\left(\frac{r^{2 n+1}}{\lambda_{1}(\operatorname{det} \Lambda)^{n}}\right) .
$$

The constant in $O(\ldots)$ depends only on $n, m$.

Proof. Suppose first that

$$
\lambda_{2}>r .
$$

Then any points $\boldsymbol{x}_{0}, \ldots, \boldsymbol{x}_{n}$ with $\left(\boldsymbol{x}_{0}, \ldots, \boldsymbol{x}_{n}\right) \in \mathcal{S}$ and $\boldsymbol{x}_{i} \in \Lambda(i=0, \ldots, n)$ have Euclidean norm $\leq r<\lambda_{2}$, and therefore are colinear. We obtain $N^{*}$ $=0$. The relation $(2.6)$ is valid since

$$
V(\mathcal{S}) / \operatorname{det} \Lambda \ll r^{2 n+2} / \operatorname{det} \Lambda<r^{2 n+1} \lambda_{2} / \operatorname{det} \Lambda \ll r^{2 n+1} / \lambda_{1}
$$

by $(2.7),(2.1)$.

Next, suppose that

$$
\lambda_{2} \leq r
$$

Let $\Lambda^{*}=\Lambda \times \ldots \times \Lambda$ in $\mathbb{R}^{2 n+2}$. Then $\operatorname{det} \Lambda^{*}=(\operatorname{det} \Lambda)^{n+1}$ and the successive minima $\lambda_{i}^{*}$ of $\Lambda^{*}$ are easily seen to be

$$
\lambda_{i}^{*}= \begin{cases}\lambda_{1} & \text { when } 1 \leq i \leq n+1 \\ \lambda_{2} & \text { when } n+1<i \leq 2 n+2 .\end{cases}
$$

We write

$$
N^{*}=N_{1}-N_{2},
$$

where $N_{1}$ is the number of $\boldsymbol{x}=\left(\boldsymbol{x}_{0}, \ldots, \boldsymbol{x}_{n}\right) \in \Lambda^{*} \cap \mathcal{S}$, and $N_{2}$ is the number of those $(n+1)$-tuples among them for which $\boldsymbol{x}_{0}, \ldots, \boldsymbol{x}_{n}$ do not span $\mathbb{R}^{2}$. We apply Lemma 1 with $l=2 n+2$ and see that

$$
N_{1}=\frac{V(\mathcal{S})}{(\operatorname{det} \Lambda)^{n+1}}+O\left(\frac{\lambda_{2} r^{2 n+1}}{(\operatorname{det} \Lambda)^{n+1}}\right)=\frac{V(\mathcal{S})}{(\operatorname{det} \Lambda)^{n+1}}+O\left(\frac{r^{2 n+1}}{\lambda_{1}(\operatorname{det} \Lambda)^{n}}\right),
$$

since $\lambda_{2 n+1}^{*}=\lambda_{2} \leq r$, and by $(2.1)$. As for $N_{2}$, it counts the point $(\mathbf{0}, \ldots, \mathbf{0})$, as well as points $\left(\boldsymbol{x}_{0}, \ldots, \boldsymbol{x}_{n}\right) \neq(\mathbf{0}, \ldots, \mathbf{0})$ with $\boldsymbol{x}_{0}, \ldots, \boldsymbol{x}_{n}$ colinear. For the latter, we lose only a factor $n+1$ if we assume that $\boldsymbol{x}_{0} \neq \mathbf{0}$, and $\boldsymbol{x}_{1}, \ldots, \boldsymbol{x}_{n}$ are multiples of $\boldsymbol{x}_{0}$. Now $\boldsymbol{x}_{0}$ lies in the disc $\mathcal{B} \subset \mathbb{R}^{2}$ of radius $r$. By Lemma 1 with $l=2$, the number of possibilities for $\boldsymbol{x}_{0} \neq \mathbf{0}$ is

$$
\left(\pi r^{2} / \operatorname{det} \Lambda\right)+O\left(1+\lambda_{2} r / \operatorname{det} \Lambda\right) \ll r^{2} / \operatorname{det} \Lambda
$$

by (2.8), and since $r^{2} \geq \lambda_{1} \lambda_{2} \gg \operatorname{det} \Lambda$ by (2.1). Each $\boldsymbol{x}_{i}(i=1, \ldots, n)$ lies in the segment $S$ of points spanned by $\boldsymbol{x}_{0}$ having Euclidean norm $\leq r$. Since $V(S)=0$, we see from Lemma 1 that the number of possibilities for each 
$\boldsymbol{x}_{i}(i=1, \ldots, n)$ is $\ll \lambda_{2} r / \operatorname{det} \Lambda$. Thus

$$
N_{2} \ll 1+\frac{\lambda_{2}^{n} r^{n+2}}{(\operatorname{det} \Lambda)^{n+1}} \ll \frac{\lambda_{2} r^{2 n+1}}{(\operatorname{det} \Lambda)^{n+1}} \ll \frac{r^{2 n+1}}{\lambda_{1}(\operatorname{det} \Lambda)^{n}}
$$

by $(2.1),(2.8)$, on noting that

$$
1 \ll\left(\lambda_{1} \lambda_{2} / \operatorname{det} \Lambda\right)^{n+1} \leq\left(\lambda_{2}^{2} / \operatorname{det} \Lambda\right)^{n+1} \leq \lambda_{2}^{n} r^{n+2} /(\operatorname{det} \Lambda)^{n+1} .
$$

The lemma follows by combining our estimates for $N_{1}$ and $N_{2}$.

3. Estimates for a given ideal class. The case $\Delta<0$. Let $K$ be a quadratic number field of discriminant $\Delta<0$. We may consider $K$ to be embedded in $\mathbb{C}$. With $\alpha \in K$ we associate the point

$$
\widehat{\alpha}=(\operatorname{Re} \alpha, \operatorname{Im} \alpha) \in \mathbb{R}^{2} .
$$

As $\alpha$ runs through the integers of $K$, then $\widehat{\alpha}$ runs through a lattice $\Lambda \subset \mathbb{R}^{2}$ of determinant $\frac{1}{2}|\Delta|^{1 / 2}$. As $\alpha$ runs through a nonzero ideal $\mathfrak{a}$ of $K$, then $\widehat{\alpha}$ runs through a lattice $\Lambda(\mathfrak{a})$ with $\operatorname{det} \Lambda(\mathfrak{a})=\frac{1}{2}|\Delta|^{1 / 2} \mathfrak{N}(\mathfrak{a})$. Denote the successive minima of $\Lambda(\mathfrak{a})$ by $\lambda_{1}(\mathfrak{a}), \lambda_{2}(\mathfrak{a})$.

Let $\mathfrak{C}$ be an ideal class of $K$. We define $\mathfrak{N}(\mathfrak{C})$ to be the minimum of $\mathfrak{N}(\mathfrak{c})$ over all integral ideals $\mathfrak{c}$ in $\mathfrak{C}$. It is well known that $\mathfrak{N}(\mathfrak{C}) \leq|\Delta|^{1 / 2}$ (see, e.g., Hecke [6, Satz 96]). The ideal class $\overline{\mathfrak{C}}$ consisting of ideals $\overline{\mathfrak{c}}$ with $\mathfrak{c} \in \mathfrak{C}$ (where the bar indicates complex conjugation) is the inverse of $\mathfrak{C}$, so that $\mathfrak{N}\left(\mathfrak{C}^{-1}\right)=\mathfrak{N}(\overline{\mathfrak{C}})=\mathfrak{N}(\mathfrak{C})$.

Now let $\mathfrak{a}$ be an ideal lying in the ideal class $\mathfrak{A}$. When $\alpha \neq 0$ lies in $\mathfrak{a}$, then $(\alpha)=\mathfrak{a} \mathfrak{b}$ with $\mathfrak{b}$ integral in $\mathfrak{A}^{-1}$, so that $|\alpha|^{2}=\mathfrak{N}(\alpha) \geq \mathfrak{N}(\mathfrak{a}) \mathfrak{N}\left(\mathfrak{A}^{-1}\right)=$ $\mathfrak{N}(\mathfrak{a}) \mathfrak{N}(\mathfrak{A})$, and

$$
\lambda_{1}(\mathfrak{a}) \geq(\mathfrak{N}(\mathfrak{a}) \mathfrak{N}(\mathfrak{A}))^{1 / 2} .
$$

Again let $\mathfrak{a}$ be in the class $\mathfrak{A}$, and write $Z_{1}(\mathfrak{a}, X)$ for the number of nonzero elements $\alpha \in \mathfrak{a}$ with $\mathfrak{N}(\alpha) \leq X \mathfrak{N}(\mathfrak{a})$.

LEMMA 4.

$$
Z_{1}(\mathfrak{a}, X)=2 \pi X /|\Delta|^{1 / 2}+O\left(X^{1 / 2} / \mathfrak{N}(\mathfrak{A})^{1 / 2}\right) .
$$

Proof. $Z_{1}(\mathfrak{a}, X)$ is the number of nonzero $\widehat{\alpha} \in \Lambda(\mathfrak{a})$ with $|\widehat{\alpha}|^{2} \leq X \mathfrak{N}(\mathfrak{a})$. By Lemma 2 with $r=(X \mathfrak{N}(\mathfrak{a}))^{1 / 2}$,

$$
Z_{1}(\mathfrak{a}, X)=(\pi X \mathfrak{N}(\mathfrak{a}) / \operatorname{det} \Lambda(\mathfrak{a}))+O\left(r / \lambda_{1}(\mathfrak{a})\right) .
$$

Substituting $\operatorname{det} \Lambda(\mathfrak{a})=\frac{1}{2}|\Delta|^{1 / 2} \mathfrak{N}(\mathfrak{a})$, the value of $r$, as well as (3.1), we obtain the desired result.

Let $n>0$ and write points in $\mathbb{R}^{2 n+2}$ as $\widehat{\boldsymbol{\alpha}}=\left(\widehat{\alpha}_{0}, \ldots, \widehat{\alpha}_{n}\right)$ with each $\widehat{\alpha}_{i} \in$ $\mathbb{R}^{2}$. With $\boldsymbol{\alpha}=\left(\alpha_{0}, \ldots, \alpha_{n}\right)$ in $K^{n+1}$ we associate the point $\widehat{\boldsymbol{\alpha}}=\left(\widehat{\alpha}_{0}, \ldots, \widehat{\alpha}_{n}\right)$. Let $\mathcal{S}$ be a compact set in $\mathbb{R}^{2 n+2}$ contained in the unit ball centered at the origin. Further suppose that $\mathcal{S}$ is of class $m$ as defined in Section 2. For 
$t>0$, let $t \mathcal{S}$ be the set of points $t \widehat{\boldsymbol{\alpha}}$ with $\widehat{\boldsymbol{\alpha}} \in \mathcal{S}$. When $\mathfrak{a}$ is a nonzero ideal in $K$, let $Z_{2}(\mathfrak{a}, \mathcal{S}, X)$ be the number of nonzero $\boldsymbol{\alpha}=\left(\alpha_{0}, \ldots, \alpha_{n}\right) \in K^{n+1}$ with each $\alpha_{i} \in \mathfrak{a}$, such that $P=\left(\alpha_{0}: \ldots: \alpha_{n}\right)$ has $\mathbb{Q}(P)=K$, and such that

$$
\widehat{\boldsymbol{\alpha}}=\left(\widehat{\alpha}_{0}, \ldots, \widehat{\alpha}_{n}\right) \in(X \mathfrak{N}(\mathfrak{a}))^{1 / 2} \mathcal{S} .
$$

LEMmA 5. When $\mathfrak{a}$ is in the ideal class $\mathfrak{A}$,

$$
Z_{2}(\mathfrak{a}, \mathcal{S}, X)=V(\mathcal{S})\left(2 X /|\Delta|^{1 / 2}\right)^{n+1}+O\left(\frac{X^{n+(1 / 2)}}{|\Delta|^{n / 2} \mathfrak{N}(\mathfrak{A})^{1 / 2}}\right) .
$$

In agreement with the convention made in the introduction, the implied constant in $O(\ldots)$ depends only on $n, m$.

Proof. $Z_{2}(\mathfrak{a}, \mathcal{S}, X)$ is the number of $\left(\widehat{\alpha}_{0}, \ldots, \widehat{\alpha}_{n}\right)$ with $(3.2)$, such that each $\widehat{\alpha}_{i} \in \Lambda(\mathfrak{a})$, and such that $\widehat{\alpha}_{0}, \ldots, \widehat{\alpha}_{n}$ span $\mathbb{R}^{2}$. We apply Lemma 3 with $\mathcal{S}$ replaced by $(X \mathfrak{N}(\mathfrak{a}))^{1 / 2} \mathcal{S}$, and with $r=(X \mathfrak{N}(\mathfrak{a}))^{1 / 2}$. We obtain

$$
Z_{2}(\mathfrak{a}, \mathcal{S}, X)=V(\mathcal{S}) \frac{(X \mathfrak{N}(\mathfrak{a}))^{n+1}}{(\operatorname{det} \Lambda(\mathfrak{a}))^{n+1}}+O\left(\frac{(X \mathfrak{N}(\mathfrak{a}))^{n+(1 / 2)}}{\lambda_{1}(\mathfrak{a})(\operatorname{det} \Lambda(\mathfrak{a}))^{n}}\right)
$$

The lemma follows after we substitute $\operatorname{det} \Lambda(\mathfrak{a})=\frac{1}{2}|\Delta|^{1 / 2} \mathfrak{N}(\mathfrak{a})$ and (3.1).

4. Estimates for a given ideal class. The case $\Delta>0$. Let $K$ be a quadratic number field with discriminant $\Delta>0$. Let $\varepsilon$ be the fundamental unit with $\varepsilon>1$, and set $R=\log \varepsilon$. Then $R \gg 1$ with an absolute implied constant. Define $t$ and $u>0$ by

$$
t=[R]+1, \quad \log u=R / t,
$$

where [ ] denotes the integer part. Then

$$
u^{t}=\varepsilon \quad \text { and } \quad 1 \ll \log u \leq 1 .
$$

With $\alpha \in K$ we associate the point

$$
\widehat{\alpha}=\left(\alpha, \alpha^{\prime}\right) \in \mathbb{R}^{2},
$$

where $\alpha^{\prime}$ is the conjugate of $\alpha$. As $\alpha$ runs through the integers of $K$, then $\widehat{\alpha}$ runs through a lattice $\Lambda \subset \mathbb{R}^{2}$ of determinant $\Delta^{1 / 2}$. As $\alpha$ runs through a nonzero ideal $\mathfrak{a}$, then $\widehat{\alpha}$ runs through a lattice $\Lambda(\mathfrak{a})$ with $\operatorname{det} \Lambda(\mathfrak{a})=$ $\Delta^{1 / 2} \mathfrak{N}(\mathfrak{a})$.

Let $v=\sqrt{u}$, so that $1 \ll \log v$ by (4.2), and

$$
v-1 \gg 1 \text {. }
$$

Let $\tau$ be the linear map $\mathbb{R}^{2} \rightarrow \mathbb{R}^{2}$ with $\tau\left(\alpha, \alpha^{\prime}\right)=\left(v^{-1} \alpha, v \alpha^{\prime}\right)$. Then $\Lambda(\mathfrak{a}, j):=\tau^{j} \Lambda(\mathfrak{a}) \quad($ for $j \in \mathbb{Z})$ is a lattice with $\operatorname{det} \Lambda(\mathfrak{a}, j)=\operatorname{det} \Lambda(\mathfrak{a})=$ $\Delta^{1 / 2} \mathfrak{N}(\mathfrak{a})$. Its first minimum is given by

$$
\lambda_{1}(\mathfrak{a}, j)=\min _{\alpha \in \mathfrak{a} \backslash\{\mathbf{0}\}}\left(v^{-2 j}|\alpha|^{2}+v^{2 j}\left|\alpha^{\prime}\right|^{2}\right)^{1 / 2} .
$$


Given $\boldsymbol{\alpha}=\left(\alpha_{0}, \ldots, \alpha_{n}\right) \in K^{n+1} \backslash\{\mathbf{0}\}$, set $\boldsymbol{\alpha}^{\prime}=\left(\alpha_{0}^{\prime}, \ldots, \alpha_{n}^{\prime}\right)$ and

$$
\psi(\boldsymbol{\alpha})=|\boldsymbol{\alpha}| /\left|\boldsymbol{\alpha}^{\prime}\right|,
$$

where $|\boldsymbol{\alpha}|=\max \left(\left|\alpha_{0}\right|, \ldots,\left|\alpha_{n}\right|\right)$. After scalar multiplication by $\varepsilon$, we have $\psi(\varepsilon \boldsymbol{\alpha})=\left|\varepsilon / \varepsilon^{\prime}\right| \psi(\boldsymbol{\alpha})=\varepsilon^{2} \psi(\boldsymbol{\alpha})$. There is a unique integer $s$ with $\varepsilon^{-1}<$ $\psi\left(\varepsilon^{s} \boldsymbol{\alpha}\right) \leq \varepsilon$. In view of the unit -1 , there are exactly two units $\eta$ such that

$$
\varepsilon^{-1}<\psi(\eta \boldsymbol{\alpha}) \leq \varepsilon
$$

The interval $\varepsilon^{-1}<x \leq \varepsilon$ is the disjoint union of the $2 t$ intervals $u^{j-1}<x$ $\leq u^{j}$ with $-t<j \leq t$.

We now consider the set $S(\mathfrak{a}, j)$ of nonzero $\left(\alpha_{0}, \ldots, \alpha_{n}\right) \in K^{n+1}$ with $\alpha_{i} \in \mathfrak{a}(0 \leq i \leq n)$ and $u^{j-1}<\psi(\boldsymbol{\alpha}) \leq u^{j}$. This set is in 1-1 correspondence with the set $\widehat{S}(\mathfrak{a}, j)$ of points $\left(\widehat{\alpha}_{0}, \ldots, \widehat{\alpha}_{n}\right) \in \mathbb{R}^{2 n+2}$ with $\widehat{\alpha}_{i} \in \Lambda(\mathfrak{a})$ $(0 \leq i \leq n)$ and with $u^{j-1}<\psi(\widehat{\boldsymbol{\alpha}}) \leq u^{j}$, where for $\widehat{\boldsymbol{\alpha}}=\left(\widehat{\alpha}_{0}, \ldots, \widehat{\alpha}_{n}\right)=$ $\left(\alpha_{0}, \alpha_{0}^{\prime}, \ldots, \alpha_{n}, \alpha_{n}^{\prime}\right)$ we set $\psi(\widehat{\boldsymbol{\alpha}})=|\boldsymbol{\alpha}| /\left|\boldsymbol{\alpha}^{\prime}\right|$ with $\boldsymbol{\alpha}=\left(\alpha_{0}, \ldots, \alpha_{n}\right)$ and $\boldsymbol{\alpha}^{\prime}=\left(\alpha_{0}^{\prime}, \ldots, \alpha_{n}^{\prime}\right)$. Let $\tau^{*}=\tau \times \ldots \times \tau$ be the map of $\mathbb{R}^{2 n+2}$ with $\tau^{*}\left(\boldsymbol{\alpha}, \boldsymbol{\alpha}^{\prime}\right)=$ $\left(v^{-1} \boldsymbol{\alpha}, v \boldsymbol{\alpha}^{\prime}\right)$, i.e., $\tau^{*}\left(\alpha_{0}, \alpha_{0}^{\prime}, \ldots, \alpha_{n}, \alpha_{n}^{\prime}\right)=\left(v^{-1} \alpha_{0}, v \alpha_{0}^{\prime}, \ldots, v^{-1} \alpha_{n}, v \alpha_{n}^{\prime}\right)$. We have $\psi\left(\tau^{*} \widehat{\boldsymbol{\alpha}}\right)=v^{-2} \psi(\widehat{\boldsymbol{\alpha}})=u^{-1} \psi(\widehat{\boldsymbol{\alpha}})$. Therefore $\widehat{\widehat{S}}(\mathfrak{a}, j):=\tau^{* j} \widehat{S}(\mathfrak{a}, j)$ consists of points $\widehat{\boldsymbol{\alpha}}=\left(\widehat{\alpha}_{0}, \ldots, \widehat{\alpha}_{n}\right)$ with

$$
\widehat{\alpha}_{i} \in \Lambda(\mathfrak{a}, j) \quad(i=0, \ldots, n) \quad \text { and } \quad u^{-1}<\psi(\widehat{\boldsymbol{\alpha}}) \leq 1 .
$$

Now let $n=0$, let $\mathfrak{a}$ be a nonzero ideal, and $-t<j \leq t$. Write $Z_{1}(\mathfrak{a}, j, X)$ for the number of nonzero $\alpha \in \mathfrak{A}$ with $\alpha \in \mathfrak{a},\left|\alpha \alpha^{\prime}\right| \leq X \mathfrak{N}(\mathfrak{a})$ and $u^{j-1}<$ $\psi(\alpha) \leq u^{j}$.

\section{LEMMA 6.}

$$
Z_{1}(\mathfrak{a}, j, X)=\left(2 R X / t \Delta^{1 / 2}\right)+O\left(X^{1 / 2} \mathfrak{N}(\mathfrak{a})^{1 / 2} / \lambda_{1}(\mathfrak{a}, j)\right) .
$$

Proof. The set of $\widehat{\alpha}=\left(\alpha, \alpha^{\prime}\right) \in \mathbb{R}^{2}$ with $\left|\alpha \alpha^{\prime}\right| \leq X \mathfrak{N}(\mathfrak{a})$ is invariant under $\tau$. Therefore $Z_{1}(\mathfrak{a}, j, X)$ is the number of $\widehat{\alpha} \in \Lambda(\mathfrak{a}, j)$ with

$$
0<\left|\alpha \alpha^{\prime}\right| \leq X \mathfrak{N}(\mathfrak{a}) \quad \text { and } \quad u^{-1}<\psi(\widehat{\alpha}) \leq 1 .
$$

These two inequalities define a set $\mathcal{S}$ in $\mathbb{R}^{2}$. For $\widehat{\alpha} \in \mathcal{S}$, we have $|\alpha| \leq\left|\alpha^{\prime}\right|<$ $u|\alpha|$, so that both $|\alpha|,\left|\alpha^{\prime}\right|<(u X \mathfrak{N}(\mathfrak{a}))^{1 / 2}$, and $\mathcal{S}$ is contained in a disc of radius $r \ll(X \mathfrak{N}(\mathfrak{a}))^{1 / 2}$. Further $\mathcal{S}$ is of some class $m \ll 1$ (in fact $m=2$ ). Although $\mathcal{S}$ is not closed, it is easily seen that Lemma 2 still applies, and we get

$$
Z_{1}(\mathfrak{a}, j, X)=(V(\mathcal{S}) / \operatorname{det} \Lambda(\mathfrak{a}, j))+O\left(r / \lambda_{1}(\mathfrak{a}, j)\right) .
$$

Since $\operatorname{det} \Lambda(\mathfrak{a}, j)=\Delta^{1 / 2} \mathfrak{N}(\mathfrak{a})$, and since, as is seen by an easy calculation, $V(\mathcal{S})=2 X \mathfrak{N}(\mathfrak{a}) \log u=2 X R \mathfrak{N}(\mathfrak{a}) / t$, the lemma follows.

Let $n>0$ and write points in $\mathbb{R}^{2 n+2}$ as $\widehat{\boldsymbol{\alpha}}=\left(\widehat{\alpha}_{0}, \ldots, \widehat{\alpha}_{n}\right)$ where each $\widehat{\alpha}_{i}=\left(\alpha_{i}, \alpha_{i}^{\prime}\right) \in \mathbb{R}^{2}$, or else as $\widehat{\boldsymbol{\alpha}}=\left(\boldsymbol{\alpha}, \boldsymbol{\alpha}^{\prime}\right)$ with $\boldsymbol{\alpha}=\left(\alpha_{0}, \ldots, \alpha_{n}\right), \boldsymbol{\alpha}^{\prime}=$ $\left(\alpha_{0}^{\prime}, \ldots, \alpha_{n}^{\prime}\right)$. With $\boldsymbol{\alpha}=\left(\alpha_{0}, \ldots, \alpha_{n}\right) \in K^{n+1}$ we associate the point $\widehat{\boldsymbol{\alpha}}=$ 
$\left(\widehat{\alpha}_{0}, \ldots, \widehat{\alpha}_{n}\right)$. Let $\mathcal{S}$ be a closed set in $\mathbb{R}^{2 n+2}$ such that the points $\widehat{\alpha}=$ $\left(\boldsymbol{\alpha}, \boldsymbol{\alpha}^{\prime}\right)$ in $\mathcal{S}$ have $|\boldsymbol{\alpha}|\left|\boldsymbol{\alpha}^{\prime}\right| \leq 2$, and that $\mathcal{S}$ is invariant under transformations $\left(\boldsymbol{\alpha}, \boldsymbol{\alpha}^{\prime}\right) \mapsto\left(t^{-1} \boldsymbol{\alpha}, t \boldsymbol{\alpha}^{\prime}\right)$ with $t>0$. For $x>1$ let $\mathcal{S}(x)$ be the intersection of $\mathcal{S}$ with $x^{-1}<\psi(\widehat{\boldsymbol{\alpha}}) \leq 1$. Points $\widehat{\boldsymbol{\alpha}} \in \mathcal{S}(x)$ have $|\boldsymbol{\alpha}|^{2} \leq 2,\left|\boldsymbol{\alpha}^{\prime}\right|^{2} \leq 2 x$, so that $\mathcal{S}(x)$ lies in a ball of radius $r \ll x^{1 / 2}$. Let $V(\mathcal{S}(x))$ be the volume of $\mathcal{S}(x)$; by the invariance property of $\mathcal{S}$ we have $V(\mathcal{S}(x))=V(\mathcal{S}(e)) \log x$. We will finally suppose that the closure of $\mathcal{S}(x)$ is of class $m$.

For a nonzero ideal $\mathfrak{a}$ and for $-t<j \leq t$, let $Z_{2}(\mathfrak{a}, j, \mathcal{S}, X)$ be the number of $\boldsymbol{\alpha}=\left(\alpha_{0}, \ldots, \alpha_{n}\right)$ with $\alpha_{i} \in \mathfrak{a}(i=0, \ldots, n)$ such that $P=\left(\alpha_{0}: \ldots: \alpha_{n}\right)$ has $\mathbb{Q}(P)=K$, and such that

$$
\widehat{\boldsymbol{\alpha}} \in(X \mathfrak{N}(\mathfrak{a}))^{1 / 2} \mathcal{S} \quad \text { and } \quad u^{j-1}<\psi(\boldsymbol{\alpha}) \leq u^{j} .
$$

\section{LEMMA 7.}

$$
Z_{2}(\mathfrak{a}, j, \mathcal{S}, X)=\frac{R V(\mathcal{S}(e))}{t}\left(\frac{X}{\Delta^{1 / 2}}\right)^{n+1}+O\left(\frac{X^{n+(1 / 2)} \mathfrak{N}(\mathfrak{a})^{1 / 2}}{\Delta^{n / 2} \lambda_{1}(\mathfrak{a}, j)}\right) .
$$

Proof. By what we have seen above, $Z_{2}(\mathfrak{a}, j, \mathcal{S}, X)$ is the same as the number of points $\widehat{\boldsymbol{\alpha}}=\left(\widehat{\alpha}_{0}, \ldots, \widehat{\alpha}_{n}\right)$ in $\Lambda(\mathfrak{a}, j) \times \ldots \times \Lambda(\mathfrak{a}, j)$ such that $\widehat{\alpha}_{0}, \ldots, \widehat{\alpha}_{n}$ span $\mathbb{R}^{2}$, and which lie in the set $\mathcal{S}^{\prime}$ defined by

$$
\left(\widehat{\alpha}_{0}, \ldots, \widehat{\alpha}_{n}\right) \in(X \mathfrak{N}(\mathfrak{a}))^{1 / 2} \mathcal{S} \quad \text { and } \quad u^{-1}<\psi(\widehat{\boldsymbol{\alpha}}) \leq 1 .
$$

$\mathcal{S}^{\prime}$ lies in a ball of radius $r \ll(X \mathfrak{N}(\mathfrak{a}))^{1 / 2}$ and has volume $V\left(\mathcal{S}^{\prime}\right)=$ $(X \mathfrak{N}(\mathfrak{a}))^{n+1}(\log u) V(\mathcal{S}(e))$. Lemma 3 gives

$$
Z_{2}(\mathfrak{a}, j, \mathcal{S}, X)=\frac{V\left(\mathcal{S}^{\prime}\right)}{(\operatorname{det} \Lambda(\mathfrak{a}, j))^{n+1}}+O\left(\frac{r^{2 n+1}}{(\operatorname{det} \Lambda(\mathfrak{a}, j))^{n} \lambda_{1}(\mathfrak{a}, j)}\right) .
$$

If we substitute our value for $V\left(\mathcal{S}^{\prime}\right)$ and $\operatorname{det} \Lambda(\mathfrak{a}, j)=\Delta^{1 / 2} \mathfrak{N}(\mathfrak{a})$, as well as the estimate for $r$, and the relation $\log u=R / t$ from (4.1), we obtain the assertion of the lemma.

Let $\mathfrak{C}$ be an ideal class. Let $\mathfrak{c}_{1}, \mathfrak{c}_{2}, \ldots$ be the integral ideals in $\mathfrak{C}$ ordered so that $\mathfrak{N}\left(\mathfrak{c}_{1}\right) \leq \mathfrak{N}\left(\mathfrak{c}_{2}\right) \leq \ldots$ We set

$$
\mathfrak{N}(\mathfrak{C})=\left(\sum_{j=1}^{2 t} \mathfrak{N}\left(\mathfrak{c}_{j}\right)^{-1 / 2}\right)^{-2} .
$$

This definition differs from the one when $\Delta<0$. It is easily seen that we still have $\mathfrak{N}\left(\mathfrak{C}^{-1}\right)=\mathfrak{N}(\overline{\mathfrak{C}})=\mathfrak{N}(\mathfrak{C})$.

Lemma 8. Let $\mathfrak{a}$ lie in the ideal class $\mathfrak{A}$. Then

$$
\sum_{j=1-t}^{t} 1 / \lambda_{1}(\mathfrak{a}, j) \ll(\mathfrak{N}(\mathfrak{a}) \mathfrak{N}(\mathfrak{A}))^{-1 / 2} .
$$

This estimate takes the place of (3.1) in the case $\Delta<0$. 
Proof. Define $\mu_{1}(\mathfrak{a}, j)$ as the minimum of $\max \left(v^{-j}|\alpha|, v^{j}\left|\alpha^{\prime}\right|\right)$ for nonzero $\alpha \in \mathfrak{a}$. Since $\lambda_{1}(\mathfrak{a}, j) \geq \mu_{1}(\mathfrak{a}, j)$, it will suffice to estimate the sum (4.7) with $\mu_{1}$ in place of $\lambda_{1}$. Pick $\alpha=\alpha(\mathfrak{a}, j)$ with

$$
\mu_{1}(\mathfrak{a}, j)=\max \left(v^{-j}|\alpha|, v^{j}\left|\alpha^{\prime}\right|\right) .
$$

We claim that for $1-t \leq j \leq t$,

$$
\varepsilon^{-2}<\psi(\alpha(\mathfrak{a}, j)) \leq \varepsilon^{2} .
$$

For if, say, $\psi(\alpha)>\varepsilon^{2}$, then

$$
v^{-j}|\alpha|>v^{-j} \varepsilon^{2}\left|\alpha^{\prime}\right| \geq v^{j}\left|\left(\varepsilon^{-1} \alpha\right)^{\prime}\right|,
$$

since $\varepsilon^{2} v^{-2 j} \geq \varepsilon^{2} v^{-2 t}=\varepsilon=\left|\left(\varepsilon^{-1}\right)^{\prime}\right|$. Therefore

$$
\max \left(v^{-j}|\alpha|, v^{j}\left|\alpha^{\prime}\right|\right) \geq v^{-j}|\alpha|>\max \left(v^{-j}\left|\varepsilon^{-1} \alpha\right|, v^{j}\left|\left(\varepsilon^{-1} \alpha\right)^{\prime}\right|\right) .
$$

By the minimal property of $\alpha(j, \mathfrak{a})$, this cannot happen for $\alpha=\alpha(j, \mathfrak{a})$. Therefore $\psi(\alpha(\mathfrak{a}, j)) \leq \varepsilon^{2}$. The lower bound in (4.8) is proved similarly.

Let $\alpha \in \mathfrak{a}$ be given with $\varepsilon^{-2}<\psi(\alpha) \leq \varepsilon^{2}$. We consider the sum

$$
\sum_{\substack{j \\ \alpha(\mathfrak{a}, j)=\alpha}}\left(\mu_{1}(\mathfrak{a}, j)\right)^{-1} \leq \sum_{j \in \mathbb{Z}} \min \left(v^{j}|\alpha|^{-1}, v^{-j}\left|\alpha^{\prime}\right|^{-1}\right) .
$$

Here $|\alpha|=v^{\xi}|\mathfrak{N}(\alpha)|^{1 / 2},\left|\alpha^{\prime}\right|=v^{-\xi}|\mathfrak{N}(\alpha)|^{1 / 2}$ for some $\xi$, so that the last sum becomes

$$
\begin{aligned}
|\mathfrak{N}(\alpha)|^{-1 / 2} \sum_{j \in \mathbb{Z}} \min \left(v^{j-\xi}, v^{\xi-j}\right) & \leq|\mathfrak{N}(\alpha)|^{-1 / 2} \cdot 2 \sum_{j=0}^{\infty} v^{-j} \\
& =(2 v /(v-1))|\mathfrak{N}(\alpha)|^{-1 / 2} \ll|\mathfrak{N}(\alpha)|^{-1 / 2},
\end{aligned}
$$

since $v-1 \gg 1$ by (4.3).

Suppose $s$ distinct numbers $\alpha_{1}, \ldots, \alpha_{s}$ occur among the $\alpha(\mathfrak{a}, j)$ where $-t<j \leq t$, so that clearly $s \leq 2 t$. Then

$$
\sum_{j=1-t}^{t} \mu_{1}(\mathfrak{a}, j)^{-1} \ll \sum_{j=1}^{s}\left|\mathfrak{N}\left(\alpha_{j}\right)\right|^{-1 / 2} .
$$

We have $\left(\alpha_{j}\right)=\mathfrak{a} \mathfrak{b}_{j}$ where $\mathfrak{b}_{j}$ is integral in $\mathfrak{A}^{-1}$. On the other hand, given $\mathfrak{b} \in \mathfrak{A}^{-1}$, there are precisely 4 elements $\alpha$ with $(\alpha)=\mathfrak{a} \mathfrak{b}$ and with $\varepsilon^{-2}<$ $\psi(\alpha) \leq \varepsilon^{2}$, because $\psi\left( \pm \varepsilon^{s} \alpha\right)=\varepsilon^{2 s} \psi(\alpha)$. Therefore, with certain distinct $\mathfrak{b}_{1}, \ldots, \mathfrak{b}_{2 t}$ in $\mathfrak{A}^{-1}$, the sum in (4.7) is

$$
\ll \mathfrak{N}(\mathfrak{a})^{-1 / 2} \sum_{j=1}^{2 t} \mathfrak{N}\left(\mathfrak{b}_{j}\right)^{-1 / 2} \leq \mathfrak{N}(\mathfrak{a})^{-1 / 2} \mathfrak{N}\left(\mathfrak{A}^{-1}\right)^{-1 / 2}=(\mathfrak{N}(\mathfrak{a}) \mathfrak{N}(\mathfrak{A}))^{-1 / 2},
$$

by the definition (4.6). 
By (4.2), by taking the sum over $j,-t<j \leq t$, in Lemmas 6,7 , and using Lemma 8, we immediately get the next two lemmas.

Lemma 9. Let $\mathfrak{a}$ be an ideal in the class $\mathfrak{A}$, and $Z_{1}(\mathfrak{a}, X)$ the number of nonzero $\alpha \in \mathfrak{a}$ with $\left|\alpha \alpha^{\prime}\right| \leq X \mathfrak{N}(\mathfrak{a})$ and $\varepsilon^{-1}<\psi(\alpha) \leq \varepsilon$. Then

$$
Z_{1}(\mathfrak{a}, X)=4 R X / \Delta^{1 / 2}+O\left(X^{1 / 2} / \mathfrak{N}(\mathfrak{A})^{1 / 2}\right) .
$$

Lemma 10. Let $n>0, \mathcal{S}$ a set in $\mathbb{R}^{2 n+2}$ as in Lemma 7 , and $\mathfrak{a}$ an ideal in the class $\mathfrak{A}$. Let $Z_{2}(\mathfrak{a}, \mathcal{S}, X)$ be the number of $\boldsymbol{\alpha}=\left(\alpha_{0}, \ldots, \alpha_{n}\right)$ with each $\alpha_{i} \in \mathfrak{a}$, with $P=\left(\alpha_{0}: \ldots: \alpha_{n}\right)$ having $\mathbb{Q}(P)=K$, and with

$$
\widehat{\boldsymbol{\alpha}} \in(X \mathfrak{N}(\mathfrak{a}))^{1 / 2} \mathcal{S} \quad \text { and } \quad \varepsilon^{-1}<\psi(\boldsymbol{\alpha}) \leq \varepsilon .
$$

Then

$$
Z_{2}(\mathfrak{a}, \mathcal{S}, X)=2 R V(\mathcal{S}(e))\left(X / \Delta^{1 / 2}\right)^{n+1}+O\left(X^{n+(1 / 2)} \Delta^{-n / 2} \mathfrak{N}(\mathfrak{A})^{-1 / 2}\right) .
$$

5. Proof of Theorem 1. Lemmas 4 and 9 may be combined to give

$$
Z_{1}(\mathfrak{a}, X)=\lambda R X /|\Delta|^{1 / 2}+O\left(X^{1 / 2} / \mathfrak{N}(\mathfrak{A})^{1 / 2}\right),
$$

where $R, \lambda$ are given by (1.4), (1.5). Note that the definitions of $Z_{1}(\mathfrak{a}, X)$ and $\mathfrak{N}(\mathfrak{A})$ are somewhat different when $\Delta<0$ and when $\Delta>0$.

Lemma 11. Let $\mathfrak{C}$ be an ideal class, and define $Z_{3}(\mathfrak{C}, X)$ to be the number of integral ideals $\mathfrak{c} \in \mathfrak{C}$ with $\mathfrak{N}(\mathfrak{c}) \leq X$. Then

$$
Z_{3}(\mathfrak{C}, X)=\lambda R X /\left(w \Delta^{1 / 2}\right)+O\left(X^{1 / 2} / \mathfrak{N}(\mathfrak{C})^{1 / 2}\right)
$$

where $w$ is the number of roots of 1 of the underlying quadratic number field $K$.

Proof. Let $\mathfrak{A}=\mathfrak{C}^{-1}$ and fix $\mathfrak{a}$ in $\mathfrak{A}$. When $\mathfrak{c} \in \mathfrak{C}$ with $\mathfrak{N}(\mathfrak{c}) \leq X$, then $\mathfrak{a c}$ is a principal ideal $(\alpha)$ with $\alpha \in \mathfrak{a}, \alpha \neq 0$, and $|\mathfrak{N}(\alpha)| \leq X \mathfrak{N}(\mathfrak{a})$. Conversely, when $\alpha \in \mathfrak{a}, \alpha \neq 0$ and $|\mathfrak{N}(\alpha)| \leq X \mathfrak{N}(\mathfrak{a})$, then $(\alpha)=\mathfrak{a} \mathfrak{c}$ with integral $\mathfrak{c} \in \mathfrak{C}$ having $\mathfrak{N}(\mathfrak{c}) \leq X$.

If $\Delta<0$, then $\alpha$ is determined by $\mathfrak{c}$ up to the $w$ roots of 1 . Thus Lemma 11 follows from Lemma 4 and the definition of $Z_{1}(\mathfrak{a}, X)$. When $\Delta>0$, we may pick $\alpha$ with $\varepsilon^{-1}<\psi(\alpha) \leq \varepsilon$, and this will determine $\alpha$ up to multiplication by \pm 1 , so that we will have $w=2$ choices for $\alpha$. Now Lemma 11 follows from Lemma 9 and the definition of $Z_{1}(\mathfrak{a}, X)$ in the case $\Delta>0$.

The proof of Theorem 1 is now easily completed by taking the sum over the ideal classes in (5.2). All that is needed is the estimate

$$
\sum_{\mathfrak{C}} \mathfrak{N}(\mathfrak{C})^{-1 / 2} \ll\left(h R \log ^{+} h R\right)^{1 / 2} .
$$

When $\Delta<0$, the sum on the left here is over $h$ terms $\mathfrak{N}\left(\mathfrak{c}_{i}\right)^{-1 / 2}$, with distinct nonzero integral ideals $\mathfrak{c}_{i}$. We may suppose that $\mathfrak{N}\left(\mathfrak{c}_{1}\right) \leq \ldots \leq \mathfrak{N}\left(\mathfrak{c}_{h}\right)$. The 
number of integral ideals $\mathfrak{c}$ with $\mathfrak{N}(\mathfrak{c})=u$ is at most $\tau(u)$, the number of positive divisors of $u$. Since

$$
\sum_{u=1}^{x} \tau(u) \sim x \log x
$$

(see [5, Theorem 315]), we may conclude that $\mathfrak{N}\left(\mathfrak{c}_{i}\right) \gg i / \log ^{+} i$. Therefore

$$
\sum_{\mathfrak{C}} \mathfrak{N}(\mathfrak{C})^{-1 / 2}=\sum_{i=1}^{h} \mathfrak{N}\left(\mathfrak{c}_{i}\right)^{-1 / 2} \ll \sum_{i=1}^{h}\left(i^{-1} \log ^{+} i\right)^{1 / 2} \ll\left(h \log ^{+} h\right)^{1 / 2} .
$$

When $\Delta>0$, each $\mathfrak{N}(\mathfrak{C})^{-1 / 2}$ is by (4.6) a sum of $2 t$ terms $\mathfrak{N}\left(\mathfrak{c}_{i}\right)^{-1 / 2}$ with distinct integral ideals $\mathfrak{c}_{i}$ in $\mathfrak{C}$. Therefore the sum in (5.3) is a sum of $2 t h$ terms $\mathfrak{N}\left(\mathfrak{c}_{i}\right)^{-1 / 2}$. By the argument used above and since $t \ll R$ by (4.1), it is

$$
\ll\left(2 t h \log ^{+}(2 t h)\right)^{1 / 2} \ll\left(R h \log ^{+} R h\right)^{1 / 2} .
$$

6. Möbius inversion. In order not to have to interrupt our main argument below, we begin with the following definition. Given a nonzero ideal $\mathfrak{b}$, let $\langle\mathfrak{b}\rangle$ be its ideal class. Given an ideal class $\mathfrak{A}$, set

$$
\mathfrak{L}_{n}(\mathfrak{A})=\sum_{\mathfrak{b}} \mathfrak{N}(\mathfrak{A}\langle\mathfrak{b}\rangle)^{-1 / 2} \mathfrak{N}(\mathfrak{b})^{-n-1 / 2},
$$

where the sum is over integral ideals $\mathfrak{b}$ of the underlying quadratic field $K$. Since there are only $h$ ideal classes, the term $\mathfrak{N}(\mathfrak{A}\langle\mathfrak{b}\rangle)^{-1 / 2}$ is bounded, and the sum will be convergent for $n>0$, which we will suppose. Incidentally, it is easily seen, but will not be used here, that $\mathfrak{N}(\mathfrak{A}\langle\mathfrak{b}\rangle)^{-1 / 2} \leq \mathfrak{N}(\mathfrak{A})^{-1 / 2} \mathfrak{N}(\mathfrak{b})^{1 / 2}$, so that when $n \geq 2$ we have

$$
\mathfrak{L}_{n}(\mathfrak{A}) \leq \mathfrak{N}(\mathfrak{A})^{-1 / 2} \sum_{\mathfrak{b}} \mathfrak{N}(\mathfrak{b})^{-n} \ll \mathfrak{N}(\mathfrak{A})^{-1 / 2} .
$$

Lemmas 5, 10 may be combined to give

$$
\begin{aligned}
& Z_{2}(\mathfrak{a}, \mathcal{S}, X) \\
& \quad=V_{0}(\mathcal{S}) R\left(X /|\Delta|^{1 / 2}\right)^{n+1}+O\left(X^{n+(1 / 2)}|\Delta|^{-n / 2} \mathfrak{N}(\mathfrak{A})^{-1 / 2}\right),
\end{aligned}
$$

where $R$ is given by (1.4), and

$$
V_{0}(\mathcal{S})= \begin{cases}2^{n+1} V(\mathcal{S}) & \text { when } \Delta<0 \\ 2 V(\mathcal{S}(e)) & \text { when } \Delta>0\end{cases}
$$

Note that the hypotheses on $\mathcal{S}$ are not the same in the cases $\Delta<0$ and $\Delta>0$. Further recall that $Z_{2}(\mathfrak{a}, \mathcal{S}, X)$ is the number of nonzero $\alpha=$ $\left(\alpha_{0}, \ldots, \alpha_{n}\right) \in K^{n+1}$ such that

(i) $\alpha_{i} \in \mathfrak{a}(i=0, \ldots, n)$,

(ii) $\mathbb{Q}(P)=K$ where $P=\left(\alpha_{0}: \ldots: \alpha_{n}\right)$, 
(iii) $\widehat{\alpha} \in(X \mathfrak{N}(\mathfrak{a}))^{1 / 2} \mathcal{S}$,

(iv) when $\Delta>0$, additionally $\varepsilon^{-1}<\psi(\boldsymbol{\alpha}) \leq \varepsilon$.

Let $Z_{4}(\mathfrak{a}, \mathcal{S}, X)$ be the number of nonzero $\alpha \in K^{n+1}$ satisfying (i'), (ii), (iii), (iv), where (i') is the condition

(i') $\alpha_{0}, \ldots, \alpha_{n}$ generate the ideal $\mathfrak{a}$.

Lemma 12. When $\mathfrak{a}$ lies in the ideal class $\mathfrak{A}$,

$$
\begin{aligned}
Z_{4}(\mathfrak{a}, \mathcal{S}, X)= & \left(V_{0}(\mathcal{S}) R / \zeta_{K}(n+1)\right)\left(X /|\Delta|^{1 / 2}\right)^{n+1} \\
& +O\left(X^{n+(1 / 2)}|\Delta|^{-n / 2} \mathfrak{L}_{n}(\mathfrak{A})\right) .
\end{aligned}
$$

Pro of. When $\alpha_{0}, \ldots, \alpha_{n}$ satisfy (i), they generate an ideal $\mathfrak{a} \mathfrak{b}$ where $\mathfrak{b}$ is integral. Then (iii) may be written as $\widehat{\alpha} \in(X / \mathfrak{N}(\mathfrak{b}))^{1 / 2} \mathfrak{N}(\mathfrak{a} \mathfrak{b})^{1 / 2} \mathcal{S}$. Therefore every $\alpha$ counted by $Z_{2}(\mathfrak{a}, \mathcal{S}, X)$ is counted by $Z_{4}(\mathfrak{a} \mathfrak{b}, \mathcal{S}, X / \mathfrak{N}(\mathfrak{b}))$ for some integral $\mathfrak{b}$, and

$$
Z_{2}(\mathfrak{a}, \mathcal{S}, X)=\sum_{\mathfrak{b}} Z_{4}(\mathfrak{a} \mathfrak{b}, \mathcal{S}, X / \mathfrak{N}(\mathfrak{b}))
$$

Let $\mu$ be the Möbius function on nonzero integral ideals of $K$, so that $\mu(\mathfrak{a} \mathfrak{b})=$ $\mu(\mathfrak{a}) \mu(\mathfrak{b})$ when $\mathfrak{a}, \mathfrak{b}$ are coprime, and $\mu(\mathfrak{p})=-1, \mu\left(\mathfrak{p}^{2}\right)=\mu\left(\mathfrak{p}^{3}\right)=\ldots=0$ when $\mathfrak{p}$ is a prime ideal. Möbius inversion gives

$$
Z_{4}(\mathfrak{a}, \mathcal{S}, X)=\sum_{\mathfrak{b}} \mu(\mathfrak{b}) Z_{2}(\mathfrak{a} \mathfrak{b}, \mathcal{S}, X / \mathfrak{N}(\mathfrak{b})) .
$$

By (6.2),

$$
\begin{aligned}
Z_{2}(\mathfrak{a} \mathfrak{b}, \mathcal{S}, X / \mathfrak{N}(\mathfrak{b}))= & V_{0}(\mathcal{S}) R\left(X / \mathfrak{N}(\mathfrak{b})|\Delta|^{1 / 2}\right)^{n+1} \\
& +O\left(X^{n+(1 / 2)}|\Delta|^{-n / 2} \mathfrak{N}(\langle\mathfrak{a} \mathfrak{b}\rangle)^{-1 / 2} \mathfrak{N}(\mathfrak{b})^{-n-1 / 2}\right) .
\end{aligned}
$$

Since $\langle\mathfrak{a} \mathfrak{b}\rangle=\mathfrak{A}\langle\mathfrak{b}\rangle$ for $\mathfrak{a} \in \mathfrak{A}$, and since $\sum_{\mathfrak{b}} \mu(\mathfrak{b}) \mathfrak{N}(\mathfrak{b})^{-n-1}=1 / \zeta_{K}(n+1)$, the lemma is a consequence of (6.4), (6.1).

7. Proof of Theorem 2. Let $\mathcal{S}$ be a closed set in $\mathbb{R}^{2 n+2}$ as described in Sections 3,4 . Thus when $\Delta<0$ we suppose that $\mathcal{S}$ is contained in the ball of radius 1 centered at the origin, and is of class $m$. We now make the further assumption that $\mathcal{S}$ contains the origin in its interior, and that $\phi(\mathcal{S}) \subseteq \mathcal{S}$ for any linear transformation $\boldsymbol{\phi}:\left(\widehat{\alpha}_{0}, \ldots, \widehat{\alpha}_{n}\right) \mapsto\left(\phi\left(\widehat{\alpha}_{0}\right), \ldots, \phi\left(\widehat{\alpha}_{n}\right)\right)$, where $\phi$ is a linear transformation of $\mathbb{R}^{2}$ which is an orthogonal map followed by a homothetic map $\widehat{\alpha} \mapsto t \widehat{\alpha}$ with $0 \leq t \leq 1$. When $\lambda \in K$ with $|\lambda| \leq 1$, then $\widehat{\alpha} \mapsto \widehat{\lambda \alpha}$ where $\alpha \in K$ comes from a map $\phi$ as above, and therefore $\widehat{\alpha} \in \mathcal{S}$ implies $(\widehat{\lambda \boldsymbol{\alpha}}) \in \mathcal{S}$. In general, when $\boldsymbol{\alpha} \in K^{n+1}$, then

$$
\widehat{\boldsymbol{\alpha}} \in \mathcal{S} \quad \text { implies }(\widehat{\lambda \boldsymbol{\alpha}}) \in|\lambda| \mathcal{S} \text {. }
$$

When $\Delta>0$, we suppose that $\mathcal{S}$ is contained in the set $|\boldsymbol{\alpha}|\left|\boldsymbol{\alpha}^{\prime}\right| \leq 2$, and it contains $\mathbf{0}$ in its interior. We will further suppose that when $\left(\boldsymbol{\alpha}, \boldsymbol{\alpha}^{\prime}\right) \in \mathcal{S}$, 
then so is $\left(t \boldsymbol{\alpha}, t^{\prime} \boldsymbol{\alpha}^{\prime}\right)$ provided $t, t^{\prime} \in \mathbb{R}$ have $\left|t t^{\prime}\right| \leq 1$. This amply yields the invariance property described in Section 4 . Moreover, when $\alpha \in K^{n+1}$ with $\widehat{\boldsymbol{\alpha}} \in \mathcal{S}$ and when $|\mathfrak{N}(\lambda)|=\left|\lambda \lambda^{\prime}\right| \leq 1$, then $(\widehat{\lambda \boldsymbol{\alpha}}) \in \mathcal{S}$. In general, $\boldsymbol{\alpha} \in K^{n+1}$ and

$$
\widehat{\boldsymbol{\alpha}} \in \mathcal{S} \quad \text { implies }(\widehat{\lambda \boldsymbol{\alpha}}) \in|\mathfrak{N}(\lambda)|^{1 / 2} \mathcal{S} \text {. }
$$

As in Section 4, we will suppose that the intersection (denoted by $\mathcal{S}(x)$ ) of $\mathcal{S}$ and $x^{-1}<\psi(\boldsymbol{\alpha}) \leq 1$ has closure of class $m$.

Given $\boldsymbol{\alpha} \in K^{n+1}$, let $H_{\infty}^{\mathcal{S}}(\boldsymbol{\alpha})$ be the least positive $t$ with $\widehat{\boldsymbol{\alpha}} \in t \mathcal{S}$. From (7.1), (7.2) we conclude that

$$
H_{\infty}^{\mathcal{S}}(\lambda \boldsymbol{\alpha})=|\mathfrak{N}(\lambda)|^{1 / 2} H_{\infty}^{\mathcal{S}}(\boldsymbol{\alpha})
$$

Again, when $\alpha \in K^{n+1}$, and $\boldsymbol{\alpha} \neq \mathbf{0}$, let $\mathfrak{a}$ be the ideal generated by $\alpha_{0}, \ldots, \alpha_{n}$, and set

$$
H^{\mathcal{S}}(\boldsymbol{\alpha})=\left(H_{\infty}^{\mathcal{S}}(\boldsymbol{\alpha})\right)^{2} / \mathfrak{N}(\mathfrak{a})
$$

By (7.3), and since $\lambda \boldsymbol{\alpha}$ induces the ideal $(\lambda) \mathfrak{a}$, it is clear that $H^{\mathcal{S}}(\lambda \boldsymbol{\alpha})=$ $H^{\mathcal{S}}(\boldsymbol{\alpha})$, so that we can define a height $H^{\mathcal{S}}(P)$ of points $P \in \mathbb{P}^{n}(K)$.

It is well known (see, e.g., [14, p. 11]) that when $\Delta<0$ the field height is $H_{K}(\boldsymbol{\alpha})=|\boldsymbol{\alpha}|^{2} / \mathfrak{N}(\mathfrak{a})$, so that $H_{K}(\boldsymbol{\alpha})=H^{\mathcal{S}_{0}^{-}}(\boldsymbol{\alpha})$ with $\mathcal{S}_{0}^{-}$the set in $\mathbb{R}^{2 n+2}$ of points $\left(\xi_{0}, \eta_{0}, \ldots, \xi_{n}, \eta_{n}\right)$ with $\xi_{i}^{2}+\eta_{i}^{2} \leq 1(i=0, \ldots, n)$. Here $V\left(\mathcal{S}_{0}^{-}\right)=$ $\pi^{n+1}$, and

$$
V_{0}\left(\mathcal{S}_{0}^{-}\right)=(2 \pi)^{n+1}=\lambda^{n+1}=\nu \lambda^{n+1} \quad(\Delta<0)
$$

by (6.3), (1.5), (1.7).

When $\Delta>0$, the field height is $H_{K}(\boldsymbol{\alpha})=|\boldsymbol{\alpha}|\left|\boldsymbol{\alpha}^{\prime}\right| / \mathfrak{N}(\mathfrak{a})=H^{\mathcal{S}_{0}^{+}}(\boldsymbol{\alpha})$, with $\mathcal{S}_{0}^{+}$the set $|\boldsymbol{\alpha}|\left|\boldsymbol{\alpha}^{\prime}\right| \leq 1$. Here $\mathcal{S}_{0}^{+}(e)$ is further restricted by $e^{-1}<|\boldsymbol{\alpha}| /\left|\boldsymbol{\alpha}^{\prime}\right| \leq$ 1 , and a computation gives $V\left(\mathcal{S}_{0}^{+}(e)\right)=\frac{1}{2}(n+1) \cdot 4^{n+1}$. Therefore

$$
V_{0}\left(\mathcal{S}_{0}^{+}\right)=(n+1) \cdot 4^{n+1}=\nu \lambda^{n+1} \quad(\Delta>0)
$$

by $(6.3),(1.5),(1.7)$.

Let $Z_{5}(K, \mathcal{S}, X)$ be the number of points $P \in \mathbb{P}^{n}(K)$ with $\mathbb{Q}(P)=K$ and $H^{\mathcal{S}}(P) \leq X$.

THEOREM 2a.

$$
\begin{aligned}
Z_{5}(K, \mathcal{S}, X)= & \frac{h R}{w \zeta_{K}(n+1)} V_{0}(\mathcal{S})\left(X /|\Delta|^{1 / 2}\right)^{n+1} \\
& +O\left(X^{n+(1 / 2)}|\Delta|^{-n / 2}\left(h R \log ^{+} h R\right)^{1 / 2}\right) .
\end{aligned}
$$

Now $N^{\prime}(K, n, X)$ is $Z_{5}\left(K, \mathcal{S}_{0}, X\right)$ with the set $\mathcal{S}_{0}=\mathcal{S}_{0}^{ \pm}$described above. Theorem 2 follows on using (7.4), (7.5).

Proof of Theorem 2a. When $P=\left(\alpha_{0}: \ldots: \alpha_{n}\right) \in \mathbb{P}^{n}(K)$, the ideal $\mathfrak{a}$ generated by $\alpha_{0}, \ldots, \alpha_{n}$ depends on $P$ up to multiplication by a principal ideal, and therefore the ideal class $\mathfrak{A}$ of $\mathfrak{a}$ depends only on $P$. Let 
$Z_{6}(\mathfrak{A}, \mathcal{S}, X)$ be the number of points $P \in \mathbb{P}^{n}(K)$ with $\mathbb{Q}(P)=K$ of height $H^{\mathcal{S}}(P) \leq X$ belonging to the class $\mathfrak{A}$.

In the class $\mathfrak{A}$ pick an ideal $\mathfrak{a}$. Then when $P$ belongs to the class $\mathfrak{A}$, we may write $P=\left(\alpha_{0}: \ldots: \alpha_{n}\right)$ where $\alpha_{0}, \ldots, \alpha_{n}$ generate $\mathfrak{a}$. We have $H^{\mathcal{S}}(P)=\left(H_{\infty}^{\mathcal{S}}(\boldsymbol{\alpha})\right)^{2} / \mathfrak{N}(\mathfrak{a})$, so that $H^{\mathcal{S}}(P) \leq X$ is the same as $H_{\infty}^{\mathcal{S}}(\boldsymbol{\alpha}) \leq$ $(X \mathfrak{N}(\mathfrak{a}))^{1 / 2}$, and this is the same as $\widehat{\alpha} \in(X \mathfrak{N}(\mathfrak{a}))^{1 / 2} \mathcal{S}$. When $\Delta<0$, then $\boldsymbol{\alpha}$ generating $\mathfrak{a}$ is determined by $P$ up to multiplication by roots of 1 , so that

$$
Z_{6}(\mathfrak{A}, \mathcal{S}, X)=\frac{1}{w} Z_{4}(\mathfrak{a}, \mathcal{S}, X) .
$$

When $\Delta>0, \boldsymbol{\alpha}$ may be chosen with $\varepsilon^{-1}<\psi(\boldsymbol{\alpha}) \leq \varepsilon$, and is then unique up to a factor \pm 1 , so that (by the definition of $Z_{4}(\mathfrak{a}, \mathcal{S}, X)$ in this case) again (7.6) holds. Now $Z_{4}(\mathfrak{a}, \mathcal{S}, X)$ may be estimated by Lemma 12.

Theorem 2 a follows by taking the sum over the ideal classes $\mathfrak{A}$. The main term is certainly correct. The error term will follow once we have shown that

$$
\sum_{\mathfrak{A}} \mathfrak{L}_{n}(\mathfrak{A}) \ll\left(h R \log ^{+} h R\right)^{1 / 2} ;
$$

here the sum is over all ideal classes $\mathfrak{A}$. But by the definition (6.1),

$$
\sum_{\mathfrak{A}} \mathfrak{L}_{n}(\mathfrak{A})=\left(\sum_{\mathfrak{A}} \mathfrak{N}(\mathfrak{A})^{-1 / 2}\right)\left(\sum_{\mathfrak{b}} \mathfrak{N}(\mathfrak{b})^{-n-1 / 2}\right) .
$$

The first factor is $\ll\left(h R \log ^{+} h R\right)^{1 / 2}$ by $(5.3)$, and the second factor is

$$
\zeta_{K}\left(n+\frac{1}{2}\right) \leq \sum_{x=1}^{\infty} \tau(x) x^{-n-1 / 2} \ll 1,
$$

where $\tau(x)$ is the number of divisors of $x$.

8. Proof of Theorem 3. Let $\mathcal{S}$ be a closed set in $\mathbb{R}^{2 n+2}$ as specified in Section 7. More precisely, write $\mathcal{S}=\mathcal{S}^{-}$if it is of the type specified for $\Delta<0$, and $\mathcal{S}=\mathcal{S}^{+}$if it is of the type specified for $\Delta>0$. Let $H^{\mathcal{S}^{+}}(P)$ [or $\left.H^{\mathcal{S}^{-}}(P)\right]$ be the height of a point $P \in \mathbb{P}^{n}(A)$ where $\mathbb{Q}(P)$ is real quadratic (with discriminant $\Delta>0$ ) [or imaginary quadratic (with $\Delta<0$ )]. With either the + or - sign, let $Z_{7}^{ \pm}\left(\mathcal{S}^{ \pm}, X\right)$ be the number of points $P \in \mathbb{P}^{n}(A)$ where $\mathbb{Q}(P)$ is quadratic with $\pm \Delta>0$ and with $H^{\mathcal{S}^{ \pm}}(P) \leq X$. In what follows, for simplicity of notation, $\mathcal{S}$ will be a set of type $\mathcal{S}^{+}$when dealing with $Z_{7}^{+}$, and of type $\mathcal{S}^{-}$when dealing with $Z_{7}^{-}$.

Theorem 3a. When $n \geq 3$, then

$$
Z_{7}^{ \pm}(\mathcal{S}, X)=c_{13}^{ \pm}(\mathcal{S}) X^{n+1}+O\left(X^{n+(1 / 2)}\right)
$$

with certain constants $c_{13}^{+}(\mathcal{S}), c_{13}^{-}(\mathcal{S})$ defined below. When $n=2$, then

$$
Z_{7}^{ \pm}(\mathcal{S}, X)=c_{14}^{ \pm}(\mathcal{S}) X^{3} \log X+O\left(X^{3} \sqrt{\log X}\right),
$$


where

$$
c_{14}^{+}(\mathcal{S})=V(\mathcal{S}(e)) /\left(2 \zeta(3)^{2}\right), \quad c_{14}^{-}(\mathcal{S})=4 V(\mathcal{S}) /\left(\pi \zeta(3)^{2}\right) .
$$

Since $\mathcal{N}^{ \pm}(2, n, X)=Z_{7}^{ \pm}\left(\mathcal{S}_{0}^{ \pm}, X\right)$, and since by what we said in $\S 7$, $V\left(\mathcal{S}_{0}^{+}(e)\right)=96, V\left(\mathcal{S}_{0}^{-}\right)=\pi^{3}$ for $n=2$, we obtain the cases $n \geq 2$ of Theorem 3 . The case $n=1$ of that theorem will be dealt with in the next section.

Proof of Theorem 3a. It will be convenient to parametrize quadratic number fields by their discriminant $\Delta$. Let $\mathcal{D}$ be the set of fundamental discriminants, i.e., the set of integers which arise as the discriminant of a quadratic number field. It is well known $([6, \S 29])$ that $\mathcal{D}=\mathcal{D}_{0} \cup \mathcal{D}_{1}$, where

$$
\begin{aligned}
& \mathcal{D}_{0}=\{\Delta=4 d \mid d \equiv 2 \text { or } 3(\bmod 4), d \text { square free }\}, \\
& \mathcal{D}_{1}=\{\Delta \mid \Delta \equiv 1(\bmod 4), \Delta \text { square free, } \Delta \neq 1\} .
\end{aligned}
$$

For $\Delta \in \mathcal{D}$ we will write $h=h(\Delta), R=R(\Delta), w=w(\Delta)$, etc., for the class number, regulator (as defined in (1.4)), number of roots of unity, etc., of the quadratic field with discriminant $\Delta$. Also, with $Z_{5}(K, \mathcal{S}, X)$ the quantity introduced in the last section, we will write $Z_{5}(\Delta, \mathcal{S}, X)=Z_{5}(K, \mathcal{S}, X)$ where $K$ is the field with discriminant $\Delta$. Now if $\mathcal{D}^{+}, \mathcal{D}^{-}$consist respectively of positive and negative elements of $\mathcal{D}$, then

$$
Z_{7}^{ \pm}(\mathcal{S}, X)=\sum_{\Delta \in \mathcal{D}^{ \pm}} Z_{5}^{ \pm}(\Delta, \mathcal{S}, X)
$$

Suppose initially that $n \geq 3$. Since, as is well known (see, e.g., [16]), $h R \ll|\Delta|^{1 / 2+\delta}$ for $\delta>0$, the sum $\sum|\Delta|^{-n / 2}\left(h R \log ^{+} h R\right)^{1 / 2}$ over $\Delta \in \mathcal{D}$ is convergent. From Theorem 2a we may infer that (8.1) holds with

$$
c_{13}^{ \pm}(\mathcal{S})=V_{0}(\mathcal{S}) \sum_{\Delta \in \mathcal{D}^{ \pm}} \frac{h(\Delta) R(\Delta)}{w(\Delta) \zeta_{\Delta}(n+1)|\Delta|^{(n+1) / 2}} .
$$

Here we used the fact that the infinite sum in the definition of $c_{13}^{ \pm}(\mathcal{S})$ is clearly convergent when $n \geq 3$.

This same sum is divergent when $n=2$. When $n=2$ we will use the fact that for a point $P \in \mathbb{P}^{n}(A)$ with $\mathbb{Q}(P)$ of degree $d$, the discriminant $\Delta$ of $\mathbb{Q}(P)$ has

$$
|\Delta| \leq d^{d} H_{K}(P)^{2 d-2}
$$

(Silverman [18, Theorem 2]). In our case, $d=2$, so that $|\Delta| \leq 4 H_{K}(P)^{2}$. The hypothesis that $\mathcal{S}$ is contained in the ball of radius 1 when $\Delta<0$, and is contained in $|\boldsymbol{\alpha}|\left|\boldsymbol{\alpha}^{\prime}\right| \leq 2$ when $\Delta>0$, implies that $H_{K}(P) \leq c_{15} H^{\mathcal{S}}(P)$. Therefore $H^{\mathcal{S}}(P) \leq X$ yields

$$
|\Delta| \leq c_{16} X^{2}
$$


Setting

$$
Y=c_{16} X^{2},
$$

and denoting the intersection of $\mathcal{D}$ or $\mathcal{D}^{ \pm}$with $|\Delta| \leq Y$ by $\mathcal{D}(Y)$ or $\mathcal{D}^{ \pm}(Y)$, we may infer from Theorem 2a that in the case $n=2$ we have

$$
Z_{7}^{ \pm}(\mathcal{S}, X)=A^{ \pm} X^{3}+O\left(B X^{5 / 2}\right),
$$

where

$$
\begin{aligned}
A^{ \pm} & =V_{0}(\mathcal{S}) \sum_{\Delta \in \mathcal{D}^{ \pm}(Y)} \frac{h R}{w(\Delta) \zeta_{\Delta}(3)|\Delta|^{3 / 2}}, \\
B & =\sum_{\Delta \in \mathcal{D}(Y)}|\Delta|^{-1}\left(h R \log ^{+} h R\right)^{1 / 2} .
\end{aligned}
$$

We first turn to the evaluation of $A^{ \pm}$. Let $\left(\frac{\Delta}{l}\right)$ be the Kronecker symbol, and

$$
L(s, \Delta)=\sum_{l=1}^{\infty}\left(\frac{\Delta}{l}\right) l^{-s}
$$

the $L$-function belonging to the quadratic field with discriminant $\Delta$. Then

$$
\zeta_{\Delta}(s)=\zeta(s) L(s, \Delta)
$$

(Hecke [6, (137)]). Further

$$
\frac{\lambda h R}{w|\Delta|^{1 / 2}}=L(1, \Delta)
$$

by $[6,(145)]$, our definition (1.5) of $\lambda$, and Hecke's definition of $\kappa[6$, p. 156]. Therefore

$$
\frac{h R}{w \zeta_{\Delta}(3)|\Delta|^{3 / 2}}=\frac{L(1, \Delta)}{\lambda \zeta(3)|\Delta| L(3, \Delta)} .
$$

In the appendix it will be shown that

$$
\sum_{\Delta \in \mathcal{D}^{ \pm}(T)} L(1, \Delta) / L(3, \Delta)=(2 \zeta(3))^{-1} T+O\left(T^{7 / 10+\delta}\right) .
$$

Partial summation gives

$$
\sum_{\Delta \in \mathcal{D}^{ \pm}(Y)} L(1, \Delta) /(L(3, \Delta)|\Delta|)=(2 \zeta(3))^{-1} \log Y+O(1) .
$$

A combination of our equations yields

$$
A^{ \pm}=\frac{V_{0}(\mathcal{S})}{2 \zeta(3)^{2} \lambda}(\log Y+O(1))=\frac{V_{0}(\mathcal{S})}{\zeta(3)^{2} \lambda} \log X+O(1)
$$

by $(8.5)$, and since $V_{0}(\mathcal{S}) \ll 1$. 
When dealing with $A^{+}$, we have $V_{0}(\mathcal{S})=2 V(\mathcal{S}(e)), \lambda=4$ by $(6.3),(1.5)$, and when dealing with $A^{-}$we have $V_{0}(\mathcal{S})=8 V(\mathcal{S}), \lambda=2 \pi$. Therefore

$$
A^{ \pm}=c_{14}^{ \pm}(\mathcal{S}) \log X+O(1)
$$

with $c_{14}^{ \pm}(\mathcal{S})$ given by $(8.3)$.

Let us turn to the quantity $B$. Since $h R \ll|\Delta|$ (in fact $\ll|\Delta|^{1 / 2+\delta}$ ),

$$
B \ll\left(\log ^{+} Y\right)^{1 / 2} \sum_{\Delta \in \mathcal{D}(Y)}|\Delta|^{-3 / 8}\left((h R)^{1 / 2}|\Delta|^{-5 / 8}\right),
$$

and by Cauchy's inequality this is

$$
\ll\left(\log ^{+} Y\right)^{1 / 2}\left(\sum_{|\Delta| \in \mathcal{D}(Y)}|\Delta|^{-3 / 4}\right)^{1 / 2}\left(\sum_{\Delta \in \mathcal{D}(Y)} h R|\Delta|^{-5 / 4}\right)^{1 / 2} .
$$

The first sum on the right hand side is $\ll Y^{1 / 4}$. On the other hand, for $T>1$ we have

$$
\sum_{\Delta \in \mathcal{D}(T)} h R \ll T^{3 / 2}
$$

(see, e.g., Siegel [16], or the discussion in our appendix), and partial summation yields

$$
\sum_{\Delta \in \mathcal{D}(Y)} h R /|\Delta|^{5 / 4} \ll Y^{1 / 4}
$$

We may conclude that

$$
B \ll Y^{1 / 4}\left(\log ^{+} Y\right)^{1 / 2} \ll X^{1 / 2}(\log X)^{1 / 2} .
$$

The estimate (8.2) now follows from (8.6), (8.8), (8.9).

9. The case $n=1$ of Theorem 3. This case is easy and is independent of what has been done above. With the exception of $(0: 1)$, every point of $\mathbb{P}^{1}$ is of the type $(1: \alpha)$. When $\alpha$ is quadratic, it satisfies a unique equation $f(\alpha)=0$, where

$$
f(x)=a x^{2}+b x+c
$$

is a polynomial in $\mathbb{Z}[x]$ with $a>0, \operatorname{gcd}(a, b, c)=1$, which is irreducible over $\mathbb{Q}$. When $\mathfrak{a}$ is the fractional ideal generated by $1, \alpha$, then it follows from Gauss' Lemma that $\mathfrak{N}(\mathfrak{a})=a^{-1}$, and therefore

$$
H_{K}(1: \alpha)=a \max (1,|\alpha|) \max \left(1,\left|\alpha^{\prime}\right|\right),
$$

where $\alpha^{\prime}$ is the conjugate of $\alpha$. The right hand side here is called the Mahler measure of $\alpha$. 
Suppose $\mathbb{Q}(\alpha)$ is imaginary quadratic. Then $c>0, b^{2}<4 a c$ and $|\alpha|=$ $\left|\alpha^{\prime}\right|$, so that $H_{K}(1: \alpha)=\max (|a|,|c|)$. Therefore $\mathcal{N}^{-}(2,1, X)$ is twice the number of irreducible polynomials $f(x)$ with

$$
0<a \leq X, \quad 0<c \leq X, \quad|b|<2 \sqrt{a c},
$$

and with $\operatorname{gcd}(a, b, c)=1$. Since there are no reducible polynomials with negative discriminant, $\mathcal{N}^{-}(2,1, X)$ is twice the number of primitive integer points $(a, b, c)$ in the region $\mathcal{R}^{-}$given by $(9.1)$; here a point is primitive if its coordinates are coprime. The region $\mathcal{R}^{-}$has volume (16/9) $X^{3}$, and it is contained in a ball of radius $\ll X$. Thus when $X \geq 1$, the number of integer points in this region is $(16 / 9) X^{3}+O\left(X^{2}\right)$. This follows, e.g., from Davenport's inequality (2.4). By Möbius inversion, the number of primitive integer points in the region is $((16 / 9) \zeta(3)) X^{3}+O\left(X^{2}\right)$. We may conclude that

$$
\mathcal{N}^{-}(2,1, X)=((32 / 9) \zeta(3)) X^{3}+O\left(X^{2}\right) .
$$

Suppose $\mathbb{Q}(\alpha)$ is real quadratic. Then $b^{2}>4 a c$ and

$$
\begin{aligned}
H_{K}(1: \alpha) & =\max \left(|a|,|c|,|a \alpha|,\left|a \alpha^{\prime}\right|\right) \\
& =\max \left(|a|,|c|, \frac{1}{2}\left|b+\sqrt{b^{2}-4 a c}\right|, \frac{1}{2}\left|b-\sqrt{b^{2}-4 a c}\right|\right) .
\end{aligned}
$$

Thus $H_{K}(\alpha) \leq X$ means that $|a| \leq X,|c| \leq X$, and $|b|+\sqrt{b^{2}-4 a c} \leq 2 X$. This last condition is the same as $b^{2}-4 a c \leq(2 X-|b|)^{2}$, or $|b| \leq X+(a c / X)$, so that

$$
0<a \leq X, \quad|c| \leq X, \quad b^{2}>4 a c, \quad|b| \leq X+(a c / X) .
$$

There are only few reducible polynomials with coefficients in this range: for if $f(x)=(u x+v)\left(u^{\prime} x+v^{\prime}\right)$, then (as is well known - in fact it follows from (10.6) below)

$$
\max (|u|,|v|) \max \left(\left|u^{\prime}\right|,\left|v^{\prime}\right|\right) \ll \max (|a|,|b|,|c|)<2 X .
$$

Given nonnegative integers $\nu, \nu^{\prime}$ with $\nu+\nu^{\prime}=[\log 2 X]$, the number of integers $u, v, u^{\prime}, v^{\prime}$ with $\max (|u|,|v|) \ll e^{\nu}, \max \left(\left|u^{\prime}\right|,\left|v^{\prime}\right|\right) \ll e^{\nu^{\prime}}$ is $\ll e^{2 \nu+2 \nu^{\prime}}$ $\ll X^{2}$. Taking the sum over pairs $\nu, \nu^{\prime}$, we obtain $\ll X^{2} \log X$ reducible polynomials. Therefore up to a summand $O\left(X^{2} \log X\right)$, our $\mathcal{N}^{+}(2,1, X)$ is twice the number of primitive integer points in the region $\mathcal{R}^{+}$given by (9.2). We obtain

$$
\mathcal{N}^{+}(2,1, X)=2 V / \zeta(3)+O\left(X^{2} \log X\right),
$$

where $V$ is the volume of $\mathcal{R}^{+}$. Write $\mathcal{R}^{+}=\mathcal{R}_{1}^{+} \cup \mathcal{R}_{2}^{+}$with $\mathcal{R}_{1}^{+}, \mathcal{R}_{2}^{+}$containing 
points with $c \leq 0$ and $c>0$, respectively. Setting $c_{1}=-c$, we have

$$
\begin{aligned}
& V\left(\mathcal{R}_{1}^{+}\right)=2 \int_{0}^{X} \int_{0}^{X}\left(X-\left(a c_{1} / X\right)\right) d a d c_{1}=(3 / 2) X^{3}, \\
& V\left(\mathcal{R}_{2}^{+}\right)=2 \int_{0}^{X} \int_{0}^{X}(X+(a c / X)-2 \sqrt{a c}) d a d c=(13 / 18) X^{3} .
\end{aligned}
$$

Therefore $V=V\left(\mathcal{R}_{1}^{+}\right)+V\left(\mathcal{R}_{2}^{+}\right)=(20 / 9) X^{3}$. The case $n=1$ of Theorem 3 follows.

10. Proof of Theorem 4. Given a nonzero quadratic form as in (1.9), with rational coefficients $a_{i j}$, let $H(f)$ be the height of its coefficient vector. Proportional forms have the same height. Let $Z_{8}(n, X)$ be the number of nonzero decomposable quadratic forms as above with height $H(f) \leq X$, where proportional forms are counted as one. As was pointed out in the introduction, when $f$ is decomposable, it determines a field $K(f)$. Let $Z_{8}^{-}(n, X)$, $Z_{8}^{+}(n, X), Z_{8}^{0}(n, X)$ respectively count only those of the forms counted by $Z_{8}(n, X)$ where $K(f)$ is imaginary quadratic, real quadratic, or the rational field.

THEOREM 4a.

$$
\begin{aligned}
Z_{8}^{ \pm}(2, X) & =c_{17}^{ \pm}(2) X^{3} \log X+O\left(X^{3} \sqrt{\log X}\right), & \\
Z_{8}^{ \pm}(n, X) & =c_{17}^{ \pm}(n) X^{n+1}+O\left(X^{n+(1 / 2)}\right) & \text { when } n \geq 3, \\
Z_{8}^{0}(n, X) & =c_{17}^{0}(n) X^{n+1} \log X+O\left(X^{n+1}\right) & \text { when } n \geq 2 .
\end{aligned}
$$

This easily implies Theorem 4 . For when $f$ has coefficients $a_{i j} \in \mathbb{Z}$ with $\left|a_{i j}\right| \leq X$, then uniquely $f=t f^{*}$ where $t$ is natural and $f^{*}$ has coprime coefficients $a_{i j}^{*} \in \mathbb{Z}$. Now

$$
H\left(f^{*}\right)=\max _{i, j}\left|a_{i j}^{*}\right|=t^{-1} \max _{i, j}\left|a_{i j}\right| \leq t^{-1} X,
$$

so that (since $Z_{8}$ counts $\pm f^{*}$ as one, but $\mathcal{Z}$ counts $\pm f$ separately)

$$
\mathcal{Z}^{ \pm}(n, X)=2 \sum_{t=1}^{\infty} Z_{8}^{ \pm}(n, X / t) .
$$

When $t \leq X$, we may apply Theorem 4 a to $Z_{8}^{ \pm}(n, X / t)$, and when $t>X$ we have $Z_{8}^{ \pm}(n, X / t)=0$. Thus, e.g., when $n=2$, we have

$$
\begin{aligned}
\mathcal{Z}^{ \pm}(2, X) & =2 c_{17}^{ \pm}(2) \sum_{t=1}^{X}(X / t)^{3} \log (X / t)+O\left(\sum_{t=1}^{X}(X / t)^{3} \sqrt{\log X}\right) \\
& =2 \zeta(3) c_{17}^{ \pm}(2) X^{3} \log X+O\left(X^{3} \sqrt{\log X}\right) .
\end{aligned}
$$


Therefore the first assertion of Theorem 4 holds with $c_{8}^{ \pm}(2)=2 \zeta(3) c_{17}^{ \pm}(2)$. The other cases of Theorem 4 follow similarly.

Proof of Theorem 4a. We begin with the quantities $Z_{8}^{ \pm}(n, X)$. Let $P, P^{\prime}$ be the pair of points associated with the quadratic form $f$, as exhibited in the introduction, so that $\mathbb{Q}(P)=\mathbb{Q}\left(P^{\prime}\right)=K(f)$ is quadratic. We may represent $P, P^{\prime}$ as $\left(\alpha_{0}: \ldots: \alpha_{n}\right),\left(\alpha_{0}^{\prime}: \ldots: \alpha_{n}^{\prime}\right)$, where $\alpha_{i}, \alpha_{i}^{\prime} \in K(f)$ and $\alpha_{i}^{\prime}$ is the conjugate of $\alpha_{i}(0 \leq i \leq n)$. Then $f$ is proportional to, and may be supposed to be equal to $l l^{\prime}$ with $l(\boldsymbol{x})=\sum_{i=0}^{n} \alpha_{i} x_{i}, l^{\prime}(\boldsymbol{x})=\sum_{i=0}^{n} \alpha_{i}^{\prime} x_{i}$. Let $\mathfrak{a}$ be the ideal generated in $K(f)$ by $\alpha_{0}, \ldots, \alpha_{n}$, and $\mathfrak{a}^{\prime}$ be the ideal generated in $K(f)$ by $\alpha_{0}^{\prime}, \ldots, \alpha_{n}^{\prime}$. Further let $\mathfrak{u}$ be the ideal generated by the coefficients $a_{i j}$ of $f$. By Gauss' Lemma, $\mathfrak{u}=\mathfrak{a a}^{\prime}$, so that with $K=K(f)$, the respective norms have $\mathfrak{N}_{\mathbb{Q}}(\mathfrak{u})^{2}=\mathfrak{N}_{K}(\mathfrak{u})=\mathfrak{N}_{K}(\mathfrak{a}) \mathfrak{N}_{K}\left(\mathfrak{a}^{\prime}\right)=\mathfrak{N}_{K}(\mathfrak{a})^{2}$. Therefore

$$
H(f)=\mathfrak{N}_{K}(\mathfrak{a})^{-1} \max _{k, j}\left|a_{k j}\right| \text {. }
$$

But

so that

$$
a_{k j}= \begin{cases}\alpha_{k} \alpha_{k}^{\prime} & \text { when } k=j, \\ \alpha_{k} \alpha_{j}^{\prime}+\alpha_{j} \alpha_{k}^{\prime} & \text { when } k \neq j\end{cases}
$$

$$
H(f)=H^{\mathcal{S}}(P)
$$

with a certain set $\mathcal{S} \subset \mathbb{R}^{2 n+2}$. Namely, when we deal with $Z_{8}^{+}$, so that $K=K(f)$ is real, then $\mathcal{S}=\mathcal{S}_{1}^{+}$, say, is defined by

$$
\begin{array}{rlrl}
\left|\alpha_{k} \alpha_{k}^{\prime}\right| & \leq 1 & & (0 \leq k \leq n), \\
\left|\alpha_{k} \alpha_{j}^{\prime}+\alpha_{j} \alpha_{k}^{\prime}\right| \leq 1 & & (0 \leq j<k \leq n) .
\end{array}
$$

Clearly when $\left(\boldsymbol{\alpha}, \boldsymbol{\alpha}^{\prime}\right) \in \mathcal{S}_{1}^{+}$and $\left|t t^{\prime}\right| \leq 1$, then also $\left(t \boldsymbol{\alpha}, t^{\prime} \boldsymbol{\alpha}^{\prime}\right) \in \mathcal{S}_{1}^{+}$. Furthermore, if $k, j$ are chosen with $|\boldsymbol{\alpha}|=\left|\alpha_{k}\right|,\left|\boldsymbol{\alpha}^{\prime}\right|=\left|\alpha_{j}^{\prime}\right|$, then when $j \neq k$,

$$
|\boldsymbol{\alpha}|\left|\boldsymbol{\alpha}^{\prime}\right|=\left|\alpha_{k}\right|\left|\alpha_{j}^{\prime}\right| \leq 1+\left|\alpha_{j} \alpha_{k}^{\prime}\right| \leq 1+\left|\alpha_{k}\right|^{-1}\left|\alpha_{j}^{\prime}\right|^{-1}=1+|\boldsymbol{\alpha}|^{-1}\left|\boldsymbol{\alpha}^{\prime}\right|^{-1},
$$

so that certainly $|\boldsymbol{\alpha}|\left|\boldsymbol{\alpha}^{\prime}\right|<2$. This is also true when $j=k$. If we deal with $Z_{8}^{-}$, so that $K=K(f)$ is imaginary quadratic, then $\alpha_{j}^{\prime}$ is the complex conjugate of $\alpha_{j}$, i.e., $\alpha_{j}^{\prime}=\bar{\alpha}_{j}$, and (10.3) says that $\left|\alpha_{k}\right| \leq 1(0 \leq k \leq n)$ and $\left|2 \operatorname{Re}\left(\alpha_{k} \bar{\alpha}_{j}\right)\right| \leq 1(0 \leq j<k \leq n)$. Writing $\alpha_{k}=\xi_{k}+i \eta_{k}$ with real $\xi_{k}$, $\eta_{k}$, we see that $(10.2)$ holds with $\mathcal{S}=\mathcal{S}_{1}^{-}$given by

$$
\begin{aligned}
\xi_{k}^{2}+\eta_{k}^{2} \leq 1 & & (0 \leq k \leq n), \\
2\left|\xi_{k} \xi_{j}+\eta_{k} \eta_{j}\right| \leq 1 & & (0 \leq j<k \leq n) .
\end{aligned}
$$

To each form $f$ there belong the two points $P, P^{\prime}$. Therefore

$$
Z_{8}^{ \pm}(n, X)=\frac{1}{2} Z_{7}^{ \pm}\left(\mathcal{S}_{1}^{ \pm}, X\right) \text {. }
$$

The first two assertions of Theorem 4a now follow from Theorem 3a. In fact, we have $c_{17}^{ \pm}(n)=\frac{1}{2} c_{13}^{ \pm}\left(\mathcal{S}_{1}^{ \pm}\right)$when $n \geq 3, c_{17}^{ \pm}(2)=\frac{1}{2} c_{14}^{ \pm}\left(\mathcal{S}_{1}^{ \pm}\right)$when $n=2$. 
We next turn to the quantity $Z_{8}^{0}(n, X)$. Our work here is independent of the rest of the paper. We may suppose that the coefficients $a_{i j}$ of $f$ are relatively prime integers. When $f$ is reducible with $K(f)=\mathbb{Q}$, then $f=l l^{\prime}$ with $l=\sum \alpha_{i} x_{i}, l^{\prime}=\sum \alpha_{i}^{\prime} x_{i}$, where $\boldsymbol{\alpha}=\left(\alpha_{0}, \ldots, \alpha_{n}\right), \boldsymbol{\alpha}^{\prime}=\left(\alpha_{0}^{\prime}, \ldots, \alpha_{n}^{\prime}\right)$ are primitive points, i.e., points with coordinates in $\mathbb{Z}$, and without common factor. Writing

$$
G\left(\boldsymbol{\alpha}, \boldsymbol{\alpha}^{\prime}\right)=\max \left(\left|\alpha_{k} \alpha_{k}^{\prime}\right|(0 \leq k \leq n) \text { and }\left|\alpha_{k} \alpha_{j}^{\prime}+\alpha_{j} \alpha_{k}^{\prime}\right|(0 \leq j<k \leq n)\right),
$$

we have to deal with pairs of primitive points $\boldsymbol{\alpha}, \boldsymbol{\alpha}^{\prime}$ with

$$
G\left(\boldsymbol{\alpha}, \boldsymbol{\alpha}^{\prime}\right) \leq X .
$$

We have seen above that $G\left(\boldsymbol{\alpha}, \boldsymbol{\alpha}^{\prime}\right) \leq 1$, which is the same as (10.3), implies $|\boldsymbol{\alpha}|\left|\boldsymbol{\alpha}^{\prime}\right|<2$, so that in general

$$
\frac{1}{2}|\boldsymbol{\alpha}|\left|\boldsymbol{\alpha}^{\prime}\right| \leq G\left(\boldsymbol{\alpha}, \boldsymbol{\alpha}^{\prime}\right) \leq 2|\boldsymbol{\alpha}|\left|\boldsymbol{\alpha}^{\prime}\right|
$$

When $\boldsymbol{\alpha}=\boldsymbol{\alpha}^{\prime}$ or $\boldsymbol{\alpha}=-\boldsymbol{\alpha}^{\prime}$, we have $G\left(\boldsymbol{\alpha}, \boldsymbol{\alpha}^{\prime}\right) \geq \frac{1}{2}|\boldsymbol{\alpha}|^{2}$, so that (10.5) gives $\left|\alpha_{i}\right| \ll X^{1 / 2}$. The number of such pairs is $\ll X^{(n+1) / 2}$, which is negligible. (They correspond to quadratic forms $f$ of rank 1.) When $\boldsymbol{\alpha}, \boldsymbol{\alpha}^{\prime}$ are not related as above, we note that the pair $\alpha, \alpha^{\prime}$ gives the same quadratic form as $\boldsymbol{\alpha}^{\prime}, \boldsymbol{\alpha}$, and again we get the same quadratic form (up to a factor \pm 1 ) if $\boldsymbol{\alpha}$ or $\boldsymbol{\alpha}^{\prime}$ is replaced by minus itself. Therefore

$$
Z_{8}^{0}(n, X)=\frac{1}{8} Z_{9}(n, X)+O\left(X^{(n+1) / 2}\right),
$$

where $Z_{9}(n, X)$ is the number of ordered pairs of primitive points $\boldsymbol{\alpha}, \boldsymbol{\alpha}^{\prime}$ with (10.5).

Now let $Z_{10}(n, X)$ be the number of (not necessarily primitive) ordered pairs of nonzero integer points $\boldsymbol{\alpha}, \boldsymbol{\alpha}^{\prime}$ with (10.5).

LEMMA 13.

$$
Z_{10}(n, X)=c_{18}(n) X^{n+1} \log X+O\left(X^{n+1}\right) .
$$

This lemma easily gives what we want: Indeed, each $\boldsymbol{\alpha}, \boldsymbol{\alpha}^{\prime}$ may uniquely be written as $\boldsymbol{\alpha}=t \boldsymbol{\beta}, \boldsymbol{\alpha}^{\prime}=t^{\prime} \boldsymbol{\beta}^{\prime}$ with $t, t^{\prime}$ natural numbers and with $\boldsymbol{\beta}, \boldsymbol{\beta}^{\prime}$ primitive; and then $G\left(\boldsymbol{\beta}, \boldsymbol{\beta}^{\prime}\right)=G\left(\boldsymbol{\alpha}, \boldsymbol{\alpha}^{\prime}\right) /\left(t t^{\prime}\right)$. Therefore

$$
Z_{10}(n, X)=\sum_{t=1}^{\infty} \sum_{t^{\prime}=1}^{\infty} Z_{9}\left(n, X /\left(t t^{\prime}\right)\right)
$$

Of course, the summands vanish when $t t^{\prime}$ is large, more precisely when $t t^{\prime}>2 X$, since $G\left(\boldsymbol{\beta}, \boldsymbol{\beta}^{\prime}\right)<1 / 2$ yields $|\boldsymbol{\beta}|\left|\boldsymbol{\beta}^{\prime}\right|<1$ by (10.6). Möbius inversion in both $t, t^{\prime}$ gives

$$
Z_{9}(n, X)=\sum_{t} \sum_{t^{\prime}} \mu(t) \mu\left(t^{\prime}\right) Z_{10}\left(n, X /\left(t t^{\prime}\right)\right),
$$


where again we may restrict to summands with $t t^{\prime} \leq 2 X$. It is an easy exercise to deduce from Lemma 13 that

$$
Z_{9}(n, X)=\left(c_{18}(n) / \zeta(n+1)^{2}\right) X^{n+1} \log X+O\left(X^{n+1}\right),
$$

which in view of (10.7) gives the last assertion of Theorem 4 a with $c_{17}^{0}(n)=$ $c_{18}(n) /\left(8 \zeta(n+1)^{2}\right)$.

Incidentally, in order to deal with $\mathcal{Z}^{0}(n, X)$ in Theorem 4 , we could have avoided the twofold inversion (10.8) (but not a simple inversion) by considering pairs $\boldsymbol{\alpha}, \boldsymbol{\alpha}^{\prime}$ where just $\boldsymbol{\alpha}$ is required to be primitive.

Finally, we turn to the proof of Lemma 13. Nonzero integer points $\boldsymbol{\alpha}$ have $|\boldsymbol{\alpha}| \geq 1$, so that $Z_{10}(n, X)$ is the number of integer points $\left(\boldsymbol{\alpha}, \boldsymbol{\alpha}^{\prime}\right)$ in the set $\mathcal{T} \subset \mathbb{R}^{2 n+2}$ given by

$$
G\left(\boldsymbol{\alpha}, \boldsymbol{\alpha}^{\prime}\right) \leq X \quad \text { and } \quad|\boldsymbol{\alpha}| \geq 1,\left|\boldsymbol{\alpha}^{\prime}\right| \geq 1 .
$$

We will estimate $Z_{10}(n, X)$ using Davenport's inequality (2.4). We will show that

$$
V(\mathcal{T})=c_{18}(n) X^{n+1} \log X+O\left(X^{n+1}\right)
$$

and

$$
V\left(\mathcal{T}^{\prime}\right) \ll X^{n+1}
$$

for the projections $\mathcal{T}^{\prime}$ of $\mathcal{T}$ on the coordinate planes of dimensions $<2 n+2$; and this clearly will yield the lemma.

In view of (10.9) and (10.6), $\mathcal{T}$ is contained in a ball of radius $\ll X$, so that (10.11) is certainly true for the projection on a plane of dimension $\leq n+$ 1. Without loss of generality it will therefore suffice to prove (10.11) when $\mathcal{T}^{\prime}$ is the orthogonal projection of $\mathcal{T}$ on the coordinate plane $\Pi(l, m)$ consisting of points $\left(\alpha_{0}, \ldots, \alpha_{l}, 0, \ldots, 0, \alpha_{0}^{\prime}, \ldots, \alpha_{m}^{\prime}, 0, \ldots, 0\right)$ with $l \geq 0, m \geq 0$. In fact, we may suppose that

$$
0 \leq l \leq m \leq n
$$

Writing $\mathcal{T}^{\prime}(l, m)$ for this projection, we will show that

$$
V\left(\mathcal{T}^{\prime}(l, m)\right) \begin{cases}=c_{19}(m) X^{m+1} \log X+O\left(X^{m+1}\right) & \text { when } l=m, \\ \ll X^{m+1} & \text { when } l<m .\end{cases}
$$

This will give both (10.11) (when $l+m<2 n$ ), as well as (10.10) (when $l=m=n)$.

Points $\left(\boldsymbol{\alpha}, \boldsymbol{\alpha}^{\prime}\right)$ in $\mathcal{T}^{\prime}(l, m)$ where $|\boldsymbol{\alpha}|<1$ or $\left|\boldsymbol{\alpha}^{\prime}\right|<1$ make up a set of volume $\ll X^{m+1}$, since $\mathcal{T}$ lies in a ball of radius $\ll X$. Such points may be neglected in the estimation of $V\left(\mathcal{T}^{\prime}(l, m)\right)$. Therefore $\mathcal{T}^{\prime}(m, m)$ may be replaced by $\mathcal{T}^{\prime \prime}(m, m)$, consisting of $\left(\boldsymbol{\alpha}, \boldsymbol{\alpha}^{\prime}\right) \in \mathbb{R}^{m+1} \times \mathbb{R}^{m+1}$ with $G\left(\boldsymbol{\alpha}, \boldsymbol{\alpha}^{\prime}\right)$ $\leq X$ and $|\boldsymbol{\alpha}| \geq 1,\left|\boldsymbol{\alpha}^{\prime}\right| \geq 1$. Points $\left(\boldsymbol{\alpha}, \boldsymbol{\alpha}^{\prime}\right) \in \mathcal{T}^{\prime}(l, m)$ certainly have $\frac{1}{2}|\boldsymbol{\alpha}|\left|\boldsymbol{\alpha}^{\prime}\right|$ $\leq X$, so that for $l<m$ we note that $\mathcal{T}^{\prime}(l, m) \subseteq \mathcal{T}^{\prime \prime}(l, m)$, consisting of $\left(\boldsymbol{\alpha}, \boldsymbol{\alpha}^{\prime}\right) \in \mathbb{R}^{l+1} \times \mathbb{R}^{m+1}$ with $\frac{1}{2}|\boldsymbol{\alpha}|\left|\boldsymbol{\alpha}^{\prime}\right| \leq X$ and $|\boldsymbol{\alpha}| \geq 1,\left|\boldsymbol{\alpha}^{\prime}\right| \geq 1$. Therefore it 
will suffice to prove $(10.13)$ with $\mathcal{T}^{\prime \prime}(l, m)$ in place of $\mathcal{T}^{\prime}(l, m)$. Here $\mathcal{T}^{\prime \prime}(l, m)$ consists of $\left(\boldsymbol{\alpha}, \boldsymbol{\alpha}^{\prime}\right)$ with

$$
F\left(\boldsymbol{\alpha}, \boldsymbol{\alpha}^{\prime}\right) \leq X, \quad|\boldsymbol{\alpha}| \geq 1,\left|\boldsymbol{\alpha}^{\prime}\right| \geq 1,
$$

where

$$
F\left(\boldsymbol{\alpha}, \boldsymbol{\alpha}^{\prime}\right)= \begin{cases}G\left(\boldsymbol{\alpha}, \boldsymbol{\alpha}^{\prime}\right) & \text { when } l=m \\ \frac{1}{2}|\boldsymbol{\alpha}|\left|\boldsymbol{\alpha}^{\prime}\right| & \text { when } l<m .\end{cases}
$$

Write $\boldsymbol{\alpha}=r \boldsymbol{\beta}, \boldsymbol{\alpha}^{\prime}=r^{\prime} \boldsymbol{\beta}^{\prime}$ where $r>0, r^{\prime}>0$ and $|\boldsymbol{\beta}|=\left|\boldsymbol{\beta}^{\prime}\right|=1$, so that $1 / 2 \leq F\left(\boldsymbol{\beta}, \boldsymbol{\beta}^{\prime}\right) \leq 2$. Let $d \boldsymbol{\beta}$ be the $l$-dimensional volume element on the cube surface $\mathcal{C}(l)$ consisting of $\boldsymbol{\beta} \in \mathbb{R}^{l+1}$ with $|\boldsymbol{\beta}|=1$. (This cube has $2(l+1)$ sides of volume $2^{l}$, so that $\int_{\mathcal{C}(l)} d \boldsymbol{\beta}=2(l+1) \cdot 2^{l}$.) We have $d \boldsymbol{\alpha}=r^{l} d r d \boldsymbol{\beta}$. Similarly, $d \boldsymbol{\alpha}^{\prime}=r^{\prime m} d r^{\prime} d \boldsymbol{\beta}^{\prime}$. In terms of the coordinates $r, r^{\prime}$, $\boldsymbol{\beta}, \boldsymbol{\beta}^{\prime}$, the set $\mathcal{T}^{\prime \prime}(l, m)$ is given by $r \geq 1, r^{\prime} \geq 1$ and $r r^{\prime} F\left(\boldsymbol{\beta}, \boldsymbol{\beta}^{\prime}\right) \leq X$. Thus when $X \geq 1$,

$$
V\left(\mathcal{T}^{\prime \prime}(l, m)\right)=\int_{\mathcal{C}(l)} d \boldsymbol{\beta} \int_{\mathcal{C}(m)} d \boldsymbol{\beta}^{\prime} \int_{1}^{X / F} r^{l} d r \int_{1}^{X /(r F)} r^{\prime m} d r^{\prime},
$$

where $F=F\left(\boldsymbol{\beta}, \boldsymbol{\beta}^{\prime}\right)$. The inner double integral is

$$
\begin{cases}\left((m+1) F^{m+1}\right)^{-1} X^{m+1} \log X+O\left(X^{m+1}\right) & \text { when } l=m, \\ \ll X^{m+1} & \text { when } l<m .\end{cases}
$$

Therefore (10.13) holds with

$$
c_{19}(m)=(m+1)^{-1} \int_{\mathcal{C}(m)} \int_{\mathcal{C}(m)} F\left(\boldsymbol{\beta}, \boldsymbol{\beta}^{\prime}\right)^{-m-1} d \boldsymbol{\beta} d \boldsymbol{\beta}^{\prime} .
$$

Appendix. Certain sums involving $L$-series. As in Section 8, let

$$
L(s, \Delta)=\sum_{n=1}^{\infty}\left(\frac{\Delta}{n}\right) n^{-s} .
$$

Here $\left(\frac{\Delta}{n}\right)$ is the Kronecker symbol, defined for $\Delta \equiv 0$ or $1(\bmod 4)$. Let $\mathcal{D}$ be the set of fundamental discriminants, and $\mathcal{D}^{+}(X), \mathcal{D}^{-}(X)$ respectively the set of numbers $\Delta \in \mathcal{D}$ with $0<\Delta \leq X$ or $0<-\Delta \leq X$. We will study sums of the type

$$
S^{ \pm}(s, a, X)=\sum_{\Delta \in \mathcal{D}^{ \pm}(X)} L(s, \Delta) / L(a, \Delta) .
$$

Our goal in this appendix will be a proof of the following

Proposition. Suppose $s=\sigma+i t, a=\alpha+i b$ with $5 / 8<\sigma<\alpha$ and $5 / 4<\alpha$. Then for $\delta>0$,

$$
S^{ \pm}(s, a, X)=c_{0}(s, a) X+O\left(X^{\max (1 / 2+\delta, 3 / 2-(4 / 5) \sigma+\delta}\right)
$$


with

$$
c_{0}(s, a)=\frac{1}{2} \zeta(2 s) \prod_{p}\left(1-p^{-2}-p^{-2 s-1}+p^{-2 s-2}-p^{-s-a}+p^{-s-a-1}\right) .
$$

Remarks. Here and below, the constants implicit in $O(\ldots)$ and in $\ll$ may depend on $\delta, \sigma$ and $\alpha$ only. The case $s=1, a=3$ yields (8.7), since $c_{0}(1,3)=1 /(2 \zeta(3))$. Presumably, our conditions on $\alpha$ and $\sigma$ could be relaxed. Our method also shows that

$$
S^{ \pm}(s, X)=\sum_{\Delta \in \mathcal{D}^{ \pm}(X)} L(s, \Delta)
$$

has $S^{ \pm}(s, X) \sim c_{0}(s) X$ with

$$
c_{0}(s)=\frac{1}{2} \zeta(2 s) \prod_{p}\left(1-p^{-2}-p^{-2 s-1}+p^{-2 s-2}\right),
$$

and with an error term as in the proposition. Sums similar to $S^{ \pm}(s, X)$ were studied by Goldfeld and Hoffstein [4]. (They take sums over $\Delta \in \mathcal{D}$ with $\Delta \equiv 1(\bmod 4)$ and $0< \pm \Delta \leq X$, and with $\Delta \equiv 0(\bmod 4)$ and $0<$ $\pm \Delta \leq 4 X$. They only require that $\sigma \geq 1 / 2$. There is a slight mistake in their constant.) Since, as already noted in Section $8, \lambda h R / w=|\Delta|^{1 / 2} L(1, \Delta)$, the sums $S^{ \pm}(1, X)$ are related to sums

$$
\sum_{\Delta \in \mathcal{D}^{ \pm}(X)} h(\Delta) R(\Delta)
$$

Asymptotic formulas for such sums, but in the context of quadratic forms, and with $\Delta$ only restricted by $\Delta \equiv 0$ or $1(\bmod 4)$, had been conjectured by Gauss, and first proved by Lipschitz [9] in the case of summation over $0<-\Delta \leq X$, and by Siegel [15] over $0<\Delta \leq X$.

Our method will follow Siegel's.

We begin with a series of lemmas.

Lemma 14. Let $\mathcal{E}$ consist of the integers which are congruent to $1,5,9,13$, 8 , or $12(\bmod 16)$. Let $\mathcal{E}^{ \pm}(Y)$ be the set of $E \in \mathcal{E}$ with $0< \pm E \leq Y$. Given natural $l$, set

$$
A_{l}^{ \pm}(Y)=\sum_{E \in \mathcal{E}^{ \pm}(Y)}\left(\frac{E}{l}\right) .
$$

Then

(i) $A_{l}^{ \pm}(Y) \ll \min \left(Y, l^{1 / 2} \log ^{+} l\right)$ when $l$ is not a square.

(ii) When $l=u^{2}$, then

$$
A_{l}^{ \pm}(Y)=u^{-1} \psi(u) \phi(u) Y+O(u),
$$


where $\phi$ is Euler's function and

$$
\psi(u)= \begin{cases}3 / 8 & \text { when } u \text { is odd } \\ 1 / 2 & \text { when } u \text { is even }\end{cases}
$$

Proof. (i) When $l$ is odd, then $\left(\frac{E}{l}\right)$ is a character of modulus $l$, and this character is nontrivial when $l$ is not a square. When $E$ runs through a finite set of consecutive integers, the corresponding sum $\sum\left(\frac{E}{l}\right)$ is $\ll l^{1 / 2} \log ^{+} l$ by the Pólya-Vinogradov inequality (see, e.g., [1, Theorem 13.15]). Since $(l, 16)=1$, the same is true when $E$ runs through a finite set of consecutive elements of an arithmetic progression with common difference 16. Since $\mathcal{E}$ consists of 6 such progressions, the assertion follows.

Now let $l$ be even. Write $\mathcal{E}=\mathcal{E}_{0} \cup \mathcal{E}_{1}$, where $\mathcal{E}_{0}$ consists of integers $\equiv 8$ or $12(\bmod 16)$, and $\mathcal{E}_{1}$ of integers $\equiv 1(\bmod 4)$. For $l$ even and $E \in \mathcal{E}_{0}$, we have $\left(\frac{E}{l}\right)=0$. We therefore may restrict ourselves to $E \in \mathcal{E}_{1}$. Write $l=l_{1} l_{2}$ where $l_{1}$ is a power of 2 , and $l_{2}$ is odd. Following Siegel we observe that

$$
\varrho_{1}(E)=\left(\frac{4 l_{1}}{E}\right)\left(\frac{E}{l_{2}}\right) \quad \text { and } \quad \varrho_{2}(E)=\left(\frac{-4 l_{1}}{E}\right)\left(\frac{E}{l_{2}}\right)
$$

are nontrivial characters $\bmod 4 l$, and that

$$
\frac{1}{2}\left(\varrho_{1}(E)+\varrho_{2}(E)\right)= \begin{cases}\left(\frac{E}{l}\right) & \text { when } E \in \mathcal{E}_{1}, \\ 0 & \text { otherwise. }\end{cases}
$$

A sum $\sum \varrho_{i}(E)(i=1,2)$, where $E$ runs through a finite set of consecutive numbers, again is $\ll l^{1 / 2} \log ^{+} l$ by Pólya-Vinogradov. The assertion follows.

(ii) When $l=u^{2}$, then $A_{l}^{ \pm}(Y)$ is the number of $E \in \mathcal{E}^{ \pm}(Y)$ with $(E, u)$ $=1$. When $u$ is odd, this is the number of integers $E$ which lie in certain 6 residue classes $(\bmod 16)$, which are coprime to $u$ and lie in the interval $0< \pm E \leq Y$. The number of such integers $E$ in an interval of length $16 u$ is $6 \phi(u)$, so that $A_{l}^{ \pm}(Y)=(6 \phi(u) / 16 u) Y+O(u)$, giving (A1). When $u$ is even, then $A_{l}^{ \pm}(Y)$ is the number of integers $E \equiv 1(\bmod 4)$ with $(E, u)=1$ lying in the interval $0< \pm E \leq Y$. The number of such integers in an interval of length $2 u$ is $\phi(u)$, so that $A_{l}^{ \pm}(Y)=(\phi(u) / 2 u) Y+O(u)$, again yielding (A1).

Lemma 15. Put $B_{l}^{ \pm}(X)=\sum_{\Delta \in \mathcal{D}^{ \pm}(X)}\left(\frac{\Delta}{l}\right)$.

(i) When $l$ is not a square,

$$
B_{l}^{ \pm}(X) \ll l^{1 / 4}\left(\log ^{+} l\right)^{1 / 2} X^{1 / 2} .
$$

(ii) When $l=u^{2}$,

$$
B_{l}^{ \pm}(X)=u^{-1} \psi(u) \phi(u)\left(\sum_{\substack{q=1 \\(2 u, q)=1}}^{\infty} \mu(q) q^{-2}\right) X+O\left(X^{1 / 2} u\right) .
$$


Proof. As in $\S 8$, write $\mathcal{D}=\mathcal{D}_{0} \cup \mathcal{D}_{1}$, where $\mathcal{D}_{0}$ consists of fundamental discriminants $\Delta \equiv 0(\bmod 4)$ (i.e., $\Delta=4 E$ with $E \equiv 2$ or $3(\bmod 4), E$ square free), and $\mathcal{D}_{1}$ consists of fundamental discriminants $\Delta \equiv 1(\bmod 4)$ (i.e., $\Delta \equiv 1(\bmod 4), \Delta$ square free, $\Delta \neq 1)$. Now

$$
\sum_{\Delta \in \mathcal{D}_{0}^{ \pm}(X)}\left(\frac{\Delta}{l}\right)=\sum_{\substack{0< \pm E \leq X / 4 \\ E \equiv 2 \text { or } 3(\bmod 4) \\ E \text { square free }}}\left(\frac{4 E}{l}\right)=\sum_{q=1}^{\sqrt{X}} \mu(q) \sum_{\substack{0< \pm E \leq X / 4 \\ E \equiv 2 \text { or } 3(\bmod 4) \\ q^{2} \mid E}}\left(\frac{4 E}{l}\right) .
$$

The outer sum is understood to be over integers $q$ in $1 \leq q \leq \sqrt{X}$. The summands have $E=q^{2} E^{\prime}$ with $q$ odd and $E^{\prime} \equiv 2$ or $3(\bmod 4)$. We clearly may restrict ourselves to summands with $(l, q)=1$. We therefore obtain

$$
\sum_{\substack{q=1 \\(2 l, q)=1}}^{\sqrt{X}} \mu(q) \sum_{\substack{0< \pm E^{\prime} \leq X /\left(4 q^{2}\right) \\ E^{\prime} \equiv 2 \text { or } 3(\bmod 4)}}\left(\frac{4 E^{\prime}}{l}\right)
$$

so that

$$
\sum_{\Delta \in \mathcal{D}_{0}^{ \pm}(X)}\left(\frac{\Delta}{l}\right)=\sum_{\substack{q=1 \\(2 l, q)=1}}^{\sqrt{X}} \mu(q) \sum_{\substack{0< \pm E \leq X / q^{2} \\ E \in \mathcal{E}_{0}}}\left(\frac{E}{l}\right) .
$$

A similar computation shows that this relation remains true if $\mathcal{D}_{0}, \mathcal{E}_{0}$ are replaced by $\mathcal{D}_{1}, \mathcal{E}_{1}$. Taking the sum we get

$$
B_{l}^{ \pm}(X)=\sum_{\substack{q=1 \\(2 l, q)=1}}^{\sqrt{X}} \mu(q) \sum_{E \in \mathcal{E}^{ \pm}\left(X / q^{2}\right)}\left(\frac{E}{l}\right) .
$$

When $l$ is not a square, the inner sum is $\ll \min \left(l^{1 / 2} \log ^{+} l, X / q^{2}\right)$ by Lemma 14 , so that we get

$$
\ll \sum_{q=1}^{\infty} \min \left(l^{1 / 2} \log ^{+} l, X / q^{2}\right) \ll X^{1 / 2} l^{1 / 4}\left(\log ^{+} l\right)^{1 / 2} .
$$

When $l=u^{2}$, the inner sum is

$$
u^{-1} \psi(u) \phi(u)\left(X / q^{2}\right)+O(u)
$$

by the same lemma. Thus

$$
B_{l}^{ \pm}(X)=u^{-1} \psi(u) \phi(u)\left(\sum_{\substack{q=1 \\(2 u, q)=1}}^{\sqrt{X}} \mu(q) q^{-2}\right) X+O\left(X^{1 / 2} u\right),
$$

from which we easily get (A2). 
We now introduce a parameter $Z>1$, to be specified later.

Lemma 16. (i) When $\sigma>0$,

$$
L(s, \Delta)=L_{1}(s, \Delta, Z)+O\left(Z^{-\sigma}|\Delta|^{1 / 2} \log ^{+}|\Delta|\right)
$$

where

$$
L_{1}(s, \Delta, Z)=\sum_{n=1}^{Z}\left(\frac{\Delta}{n}\right) n^{-s} .
$$

(ii) When $a=\alpha+i b$, with $\alpha>1$, then $|L(a, \Delta)| \gg 1$.

Proof. (i) We may suppose that $Z$ is an integer.

$$
L(s, \Delta)-L_{1}(s, \Delta, Z)=\sum_{n>Z}\left(\frac{\Delta}{n}\right) n^{-s}=\sum_{n>Z}\left(s_{n}-s_{n-1}\right) n^{-s}
$$

with

$$
s_{n}:=\sum_{j=1}^{n}\left(\frac{\Delta}{j}\right) \ll|\Delta|^{1 / 2} \log ^{+}|\Delta|
$$

by Pólya-Vinogradov. We get

$$
\begin{aligned}
L(s, \Delta)-L_{1}(s, \Delta, Z) & =\sum_{n>Z} s_{n}\left(n^{-s}-(n+1)^{-s}\right)-s_{Z}(Z+1)^{-s} \\
& \ll|\Delta|^{1 / 2}\left(\log ^{+}|\Delta|\right)\left(\left(\sum_{n>Z} n^{-\sigma-1}\right)+Z^{-\sigma}\right) \\
& \ll Z^{-\sigma}|\Delta|^{1 / 2} \log ^{+}|\Delta| .
\end{aligned}
$$

(ii) follows from the product formula

$$
|L(a, \Delta)|=\prod_{p}\left|1-\left(\frac{\Delta}{p}\right) p^{-a}\right|^{-1} \geq \prod_{p}\left(1+p^{-\alpha}\right)^{-1} \gg 1 .
$$

We now turn to the proof of the proposition. By Lemma 16,

$$
S^{ \pm}(s, a, X)=\sum_{\Delta \in \mathcal{D}^{ \pm}(X)} \frac{L(s, \Delta, Z)}{L(a, \Delta)}+O\left(Z^{-\sigma} \sum_{\Delta=-X}^{X}|\Delta|^{1 / 2} \log ^{+} \Delta\right),
$$

so that

$$
S^{ \pm}(s, a, X)=S_{1}^{ \pm}(s, a, X, Z)+O\left(Z^{-\sigma} X^{3 / 2} \log X\right)
$$

where (in view of $L(a, \Delta)^{-1}=\sum_{m}\left(\frac{\Delta}{m}\right) \mu(m) m^{-a}$ ),

$$
\text { (A4) } S_{1}^{ \pm}(s, a, X, Z)=\sum_{\Delta \in \mathcal{D}^{ \pm}(X)}\left(\sum_{n=1}^{Z}\left(\frac{\Delta}{n}\right) n^{-s}\right)\left(\sum_{m=1}^{\infty}\left(\frac{\Delta}{m}\right) \mu(m) m^{-a}\right)
$$




$$
=\sum_{m=1}^{\infty} \mu(m) m^{-a} \sum_{n=1}^{Z} n^{-s} \sum_{\Delta \in \mathcal{D}^{ \pm}(X)}\left(\frac{\Delta}{m n}\right) .
$$

When $m n$ is not a square, the inner sum is $\ll X^{1 / 2}(m n)^{1 / 4}\left(\log ^{+} m n\right)^{1 / 2}$ by Lemma 15 . Therefore the terms with $m n$ not a square contribute

$$
\ll X^{1 / 2}\left(\sum_{m=1}^{\infty} m^{1 / 4-\alpha}\left(\log ^{+} m\right)^{1 / 2}\right)\left(\sum_{n=1}^{Z} n^{1 / 4-\sigma}\left(\log ^{+} n\right)^{1 / 2}\right),
$$

and since $\alpha>5 / 4$, this is

$$
\ll X^{1 / 2} \max \left(1, Z^{5 / 4-\sigma}\right)\left(\log ^{+} Z\right)^{3 / 2} .
$$

Thus

$$
\begin{aligned}
S_{1}^{ \pm}(s, a, X, Z)= & S_{2}^{ \pm}(s, a, X, Z) \\
& +O\left(X^{1 / 2} \max \left(1, Z^{5 / 4-\sigma}\right)\left(\log ^{+} Z\right)^{3 / 2}\right),
\end{aligned}
$$

where $S_{2}^{ \pm}(s, a, X, Z)$ is the sum of the terms where $m n$ is a square.

When $m n=u^{2}$, the inner sum on the right hand side of (A4) is again estimated by Lemma 15 . We have

$$
u^{-1} m^{-a} n^{-s}=u^{-2 s-1} m^{s-a}, \quad u m^{-a} n^{-s}=u^{-2 s+1} m^{s-a},
$$

so that

$$
S_{2}^{ \pm}(s, a, X, Z)=X S_{3}(s, a, Z)+O\left(X^{1 / 2} S_{3}^{*}(s, a, Z)\right),
$$

where

$$
\begin{aligned}
& S_{3}(s, a, Z)=\sum_{m=1}^{\infty} \mu(m) m^{s-a} \sum_{\substack{u=1 \\
m \mid u^{2}}}^{\sqrt{m Z}} \psi(u) \phi(u) u^{-2 s-1} \sum_{\substack{q=1 \\
(2 u, q)=1 \\
(\sqrt{2})}}^{\infty} \mu(q) q^{-2}, \\
& S_{3}^{*}(s, a, Z)=\sum_{m=1}^{\infty} m^{\sigma-\alpha} \sum_{\substack{u=1 \\
m \mid u^{2}}}^{\sqrt{m Z}} u^{-2 \sigma+1} \ll \sum_{u=1}^{\infty} u^{1-2 \sigma} \sum_{\substack{m \mid u^{2} \\
m \geq u^{2} / Z}} m^{\sigma-\alpha} .
\end{aligned}
$$

The number of divisors of $u^{2}$ is $\ll u^{\delta}$ for $\delta>0$, so that the inner sum here is $\ll u^{\delta} \min \left(1,\left(Z / u^{2}\right)^{\alpha-\sigma}\right)$, since $\alpha \geq \sigma$. Recalling that $\alpha>1$, and choosing $\delta$ sufficiently small, we get

$$
\begin{aligned}
S_{3}^{*}(s, a, Z) & \ll \sum_{u \leq \sqrt{Z}} u^{1-2 \sigma+\delta}+Z^{\alpha-\sigma} \sum_{u>\sqrt{Z}} u^{1-2 \alpha+\delta} \\
& \ll \max \left(1, Z^{1-\sigma+\delta}\right) .
\end{aligned}
$$


It remains for us to deal with $S_{3}(s, a, Z)$. Since

$$
\sum_{u>\sqrt{m Z}} \psi(u) \phi(u) u^{-2 s-1} \ll \sum_{u>\sqrt{m Z}} u^{-2 \sigma} \ll(m Z)^{1 / 2-\sigma},
$$

and since $\sum_{m} m^{1 / 2-\alpha} \ll 1$, we have

$$
S_{3}(s, a, Z)=c_{0}(s, a)+O\left(Z^{1 / 2-\sigma}\right)
$$

with

$$
c_{0}(s, a)=\sum_{u=1}^{\infty} \psi(u) \phi(u) u^{-2 s-1} \sum_{\substack{q=1 \\(2 u, q)=1}}^{\infty} \mu(q) q^{-2} \sum_{m \mid u} \mu(m) m^{s-a} .
$$

Combining (A3), (A5), (A6), (A7), (A8) we obtain

$$
\begin{aligned}
S^{ \pm}(s, a, X)= & c_{0}(s, a) X \\
& +O\left(Z^{-\sigma} X^{3 / 2+\delta}+X^{1 / 2} Z^{\delta} \max \left(1, Z^{5 / 4-\sigma}\right)+X Z^{1 / 2-\sigma}\right) .
\end{aligned}
$$

We now choose $Z=X^{4 / 5}$ to obtain the estimate of the proposition.

To evaluate $c_{0}(s, a)$ we note that

$$
\sum_{\substack{q=1 \\(2 u, q)=1}}^{\infty} \mu(q) q^{-2}=\zeta(2)^{-1} \prod_{p \mid 2 u}\left(1-p^{-2}\right)^{-1}=\zeta(2)^{-1} \varrho(u) \prod_{p \mid u}\left(1-p^{-2}\right)^{-1},
$$

where $\varrho(u)=1$ when $u$ is even, $\varrho(u)=4 / 3$ when $u$ is odd. Note that $\psi(u) \varrho(u)=1 / 2$ always. Therefore

$$
c_{0}(s, a)=(2 \zeta(2))^{-1} \sum_{u=1}^{\infty} \phi(u) u^{-2 s-1}\left(\prod_{p \mid u}\left(1-p^{-2}\right)^{-1}\left(1-p^{s-a}\right)\right) .
$$

The function in $u$ behind the $\sum$ symbol is multiplicative, so that

$$
\begin{aligned}
c_{0}(s, a)= & (2 \zeta(2))^{-1} \prod_{p}\left(1+\left(1-p^{-2}\right)^{-1}\left(1-p^{s-a}\right)\left(\sum_{\nu=1}^{\infty} \phi\left(p^{\nu}\right) / p^{\nu(2 s+1)}\right)\right) \\
= & (2 \zeta(2))^{-1} \\
& \times \prod_{p}\left(1+\left(1-p^{-2}\right)^{-1}\left(1-p^{-(a-s)}\right)\left(1-p^{-2 s}\right)^{-1}(p-1) p^{-2 s-1}\right) \\
= & \frac{1}{2} \zeta(2 s) \prod_{p}\left(\left(1-p^{-2}\right)\left(1-p^{-2 s}\right)+\left(1-p^{-(a-s)}\right)(p-1) p^{-2 s-1}\right) \\
= & \frac{1}{2} \zeta(2 s) \prod_{p}\left(1-p^{-2}-p^{-2 s-1}+p^{-2 s-2}-p^{-a-s}+p^{-a-s-1}\right) .
\end{aligned}
$$




\section{References}

[1] T. M. Apostol, Introduction to Analytic Number Theory, Springer, New York, 1976.

[2] J. W. S. Cassels, An Introduction to the Geometry of Numbers, Grundlehren Math. Wiss. 99, Springer, 1959.

[3] H. Davenport, On a principle of Lipschitz, J. London Math. Soc. 26 (1951), 179-183.

[4] D. Goldfeld and J. Hoffstein, Eisenstein series of $\frac{1}{2}$-integral weight and the mean value of real Dirichlet L-series, Invent. Math. 80 (1985), 185-208.

[5] G. H. Hardy and E. M. Wright, An Introduction to the Theory of Numbers, 3rd ed., Clarendon Press, Oxford, 1954.

[6] E. Hecke, Vorlesungen über die Theorie der algebraischen Zahlen, Chelsea, 1948.

[7] Y. R. Katznelson, Asymptotics for singular integral matrices in convex domains and applications, Ph.D. Dissertation, Stanford Univ., 1991.

[8] S. Lang, Fundamentals of Diophantine Geometry, Springer, 1983.

[9] R. Lipschitz, Sitzungsber. Akad. Berlin, 1865, 174-185.

[10] D. G. Northcott, An inequality in the theory of arithmetic on algebraic varieties, Proc. Cambridge Philos. Soc. 45 (1949), 502-509 and 510-518.

[11] S. H. Schanuel, Heights in number fields, Bull. Soc. Math. France 107 (1979), 433-449.

[12] W. M. Schmidt, Diophantine Approximations and Diophantine Equations, Lecture Notes in Math. 1467, Springer, 1991.

[13] - Northcott's theorem on heights, I. A general estimate, Monatsh. Math. 115 (1993), 169-181.

[14] J.-P. Serre, Lectures on the Mordell-Weil Theorem, Vieweg, Braunschweig, 1988.

[15] C. L. Siegel, The average measure of quadratic forms with given determinant and signature, Ann. of Math. 45 (1944), 667-685.

[16] —, Abschätzung von Einheiten, Nachr. Akad. Wiss. Göttingen, Math.-Phys. Kl. 1969, 71-86.

[17] - Lectures on the Geometry of Numbers, rewritten by K. Chandrasekharan, Springer, 1988.

[18] J. Silverman, Lower bounds for height functions, Duke Math. J. 51 (1984), 395403.

DEPARTMENT OF MATHEMATICS

UNIVERSITY OF COLORADO

BOULDER, COLORADO 80309-0395

U.S.A. 